\title{
QPX7728, An Ultra-Broad-Spectrum B-Lactamase Inhibitor for Intravenous and Oral Therapy: Overview of Biochemical and Microbiological Characteristics
}

\author{
Olga Lomovskaya ${ }^{1 *}$, Ruslan Tsivkovski ${ }^{1}$, Dongxu Sun ${ }^{1}$, Raja Reddy', Maxim Totrov' ${ }^{2}$, \\ Scott Hecker ${ }^{1}$, David Griffith ${ }^{1}$, Jeffery Loutit ${ }^{1}$ and Michael Dudley ${ }^{1}$ \\ ${ }^{1}$ Qpex Biopharma, Inc., San Diego, CA, United States, ${ }^{2}$ Molsoft, LLC, San Diego, CA, United States
}

\section{OPEN ACCESS}

Edited by:

Krisztina M. Papp-Wallace, Louis Stokes Cleveland VA Medical

Center, United States

Reviewed by: Mariagrazia Perilli,

University of L'Aquila, Italy

Sergei Vakulenko,

University of Notre Dame,

United States

Philip Hinchliffe,

University of Bristol, United Kingdom

*Correspondence:

Olga Lomovskaya olomovskaya@qpexbio.com

Specialty section: This article was submitted to Antimicrobials, Resistance and Chemotherapy,

a section of the journal Frontiers in Microbiology

Received: 19 April 2021 Accepted: 31 May 2021 Published: 05 July 2021

Citation:

Lomovskaya O, Tsivkovski R, Sun D, Reddy R, Totrov M, Hecker S, Griffith D, Loutit J and Dudley $M$ (2021) QPX7728, An

Ultra-Broad-Spectrum B-Lactamase Inhibitor for Intravenous and Oral Therapy: Overview of Biochemical and Microbiological Characteristics.

Front. Microbiol. 12:697180. doi: 10.3389/fmicb.2021.697180
QPX7728 is a novel $\beta$-lactamase inhibitor (BLI) that belongs to a class of cyclic boronates. The first member of this class, vaborbactam, is a BLI in the recently approved Vabomere (meropenem-vaborbactam). In this paper we provide the overview of the biochemical, structural and microbiological studies that were recently conducted with QPX7728. We show that QPX7728 is an ultra-broad-spectrum $\beta$-lactamase inhibitor with the broadest spectrum of inhibition reported to date in a single BLI molecule; in addition to potent inhibition of clinically important serine $\beta$-lactamases, including Class $A$ and $D$ carbapenemases from Enterobacterales and notably, diverse Class D carbapenemases from Acinetobacter, it also inhibits many metallo $\beta$-lactamases. Importantly, it is minimally affected by general intrinsic resistance mechanisms such as efflux and porin mutations that impede entry of drugs into gram-negative bacteria. QPX7728 combinations with several intravenous (IV) $\beta$-lactam antibiotics shows broad coverage of Enterobacterales, Acinetobacter baumannii and Pseudomonas aeruginosa, including strains that are resistant to other IV $\beta$-lactam-BLI combinations, e.g., ceftazidime-avibactam, ceftolozane-tazobactam, meropenem-vaborbactam and imipenem-relebactam that were recently approved for clinical use. Based on studies with $P$. aeruginosa, different partner $\beta$-lactams in combination with QPX7728 may be optimal for the coverage of susceptible organisms. This provides microbiological justification for a stand-alone BLI product for co-administration with different $\beta$-lactams. QPX7728 can also be delivered orally; thus, its ultra-broad $\beta$-lactamase inhibition spectrum and other features could be also applied to oral QPX7728-based combination products. Clinical development of QPX7728 has been initiated.

Keywords: QPX7728, beta-lactamase inhibitor, carbapenemase, metallo $\beta$-lactamase, CRE, Acinetobacter, Pseudomonas

\section{INTRODUCTION}

Development of $\beta$-lactamase inhibitors (BLIs) in combination with $\beta$-lactams continues to be a powerful strategy to restore potency of $\beta$-lactams against $\beta$-lactamase-producing bacteria, thus extending clinical utility of this important class of antibiotics (Bush, 2018; Bush and Bradford, 2019; Ho et al., 2019; Papp-Wallace, 2019). Recent approval of four BL/BLI combinations 
represents significant progress in the successful development of new IV agents to address infections due to gram-negative pathogens (Talbot et al., 2019) identified by both the Centers for Disease Control (CDC) and the World Health Organization (WHO) as urgent or serious threats (CDC, 2019) for which new agents are critically needed (WHO, 2017). However, while new BLIs, avibactam, relebactam, and vaborbactam, have a much broader spectrum of $\beta$-lactamase inhibition compared to clavulanic acid, tazobactam and sulbactam, they have no activity against metallo $\beta$-lactamases; consequently, new BL/BLI combinations have no utility in treatment of infections due to MBL-producing strains of Enterobacterales unless they are coadministered with a monobactam like aztreonam (Bush and Bradford, 2019; Papp-Wallace, 2019). Of note, recent reports have demonstrated an increasing number of CRE producing the New Delhi metallo- $\beta$-lactamase (NDM-1), notably in COVID19 patients (Nori et al., 2020; Porretta et al., 2020). In addition, none of the recently approved BLIs have activity against Class $\mathrm{D}$ carbapenemases from Acinetobacter baumannii and thus new $\mathrm{BL} / \mathrm{BLI}$ combinations cannot be used to treat infections due to carbapenem-resistant Acinetobacter.

The increase in drug-resistant infections is not limited to hospital settings; multi-drug resistance to existing oral antibiotics (fluoroquinolones, trimethoprim-sulfamethoxazole, and $\beta$-lactams) is on the rise in community settings as well (Calzi et al., 2016; Mazzariol et al., 2017; Trautner, 2018; Critchley et al., 2019). Unfortunately, none of the BL/BLI combinations that recently became available for the IV treatment of gram-negative infections due to CRE and ESBL-producing Enterobacterales are orally bioavailable (Talbot et al., 2019).

Prompted by this ongoing unmet clinical need and encouraged by our experience with cyclic boronate class that gave rise to the first FDA-approved BLI vaborbactam from this class, we continued to optimize the pharmacophore associated with inhibition of $\beta$-lactamases. The main objective was to expand the spectrum of $\beta$-lactamase inhibition of cyclic boronic acid-based BLIs to include both serine and metallo $\beta$ lactamases. In addition, we explored structures that could achieve oral bioavailability for use in combination with oral $\beta$-lactams. Although two different molecules were originally envisioned, the SAR converged with the discovery of QPX7728 which allowed us to achieve both target profiles in a single molecule.

\section{THE DISCOVERY PATH FROM VABORBACTAM TO ULTRA-BROAD-SPECTRUM QPX7728 WAS A COMBINATION OF RATIONAL STRATEGIES AND UNEXPECTED BREAKTHROUGHS}

Vaborbactam, the first boronic BLI approved as a combination product with meropenem, was specifically optimized for inhibition of KPC (Hecker et al., 2015). Its potency is ca. 1050 -fold less against other class A or class C beta lactamases compared to KPC enzymes (Tsivkovski and Lomovskaya,
2019). It has no activity against Class $D$ or Class B $\beta$ lactamases. Soon after vaborbactam was introduced into clinical development, our discovery team began to work on the next generation of boronic BLIs starting with bicyclic amide series of compounds. This effort is described in detail by Hecker et al. (2020). Earlier studies demonstrated that related compounds possessed broad-spectrum inhibition of serine $\beta$-lactamases (Ness et al., 2000) and our own modeling indicated that broadening of the spectrum to include metallo enzymes (MBLs) was possible. Indeed, the amide series represented by RPX7323 (Figure 1) were capable of potently inhibiting serine enzymes from classes A-D and there were analogs that afforded weak activity against metallo enzymes (MBLs, Class B).

The first real breakthrough came from a completely opportunistic discovery of bicyclic thioether series of compounds, as exemplified by RPX7546 (Figure 1), in which the amide linkage is replaced by a thio linkage (Reddy et al., 2014, 2016). The very first sulfur-linked compound in this series was prepared primarily because it was easy to synthesize. This replacement unexpectedly achieved improved potency against MBLs. Further optimization centered around gaining activity against Class D carbapenemases from Acinetobacter (e.g., OXA-23/OXA-24/OXA-58).

The next breakthrough came with the discovery of RPX7610 (Figure 1), a compound that lacked a substituent next to boron: it achieved potent inhibition of all target $\beta$-lactamases and also appeared to be minimally affected by efflux and porin mutations. Ironically, this unsubstituted analog was prepared as a model compound for BLIs with a heteroatom in the aryl ring; its potency and spectrum were unexpected. Advanced evaluation of this compound identified, however, its metabolic liability. This called for a rational approach to identify moieties that would confer metabolic stability but would not be detrimental for the achieved advantageous characteristics of RPX7610. This effort culminated in the discovery of QPX7728 (Hecker et al., 2020).

\section{QPX7728 With High Potency Inhibits Multiple Serine and Metallo- $\beta$-Lactamases}

The potency of inhibition of several purified $\beta$-lactamases from structural Classes A, C and D and MBLs from Class B by QPX7728 was studied by Tsivkovski et al. (2020) (Table 1). $\mathrm{IC}_{50}$ values were in low $\mathrm{nM}$ range for class A KPC-2, CTXM-15, SHV-12, TEM-43 and in double digit nM range for Class C P99. QPX7728 also inhibited with high potency ( $\mathrm{IC}_{50}$ in low $\mathrm{nM}$ range) all tested Class $\mathrm{D}$ carbapenemases, such as OXA-48 from Enterobacterales and, importantly, OXA-23 from A. baumannii.

Notably, QPX7728 inhibited clinically important MBLs, VIM1 and NDM-1 with double-digit nM potency. Somewhat lower inhibitory potency $\left(\mathrm{IC}_{50}\right.$ in $\mu \mathrm{M}$ range) was observed for IMP MBLs (Table 1).

The strain of $P$. aeruginosa PAM1154 lacks the major efflux pump MexAB-OprM and was used by Lomovskaya et al. (2020b) 


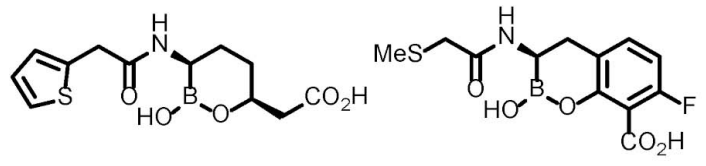

Vaborbactam

RPX7323

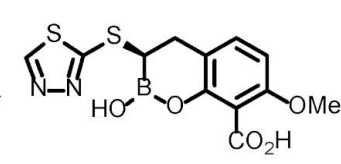

RPX7546

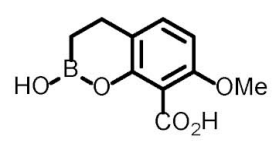

RPX7610

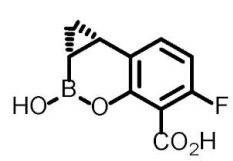

QPX7728

FIGURE 1 | Evolution of boronic $\beta$-lactamase inhibitors.

TABLE 1 | Potency $\left(\mathrm{IC}_{50}\right.$, nM) of $\beta$-lactamases inhibition by QPX7728 compared to other BLIs.

\begin{tabular}{|c|c|c|c|c|c|c|}
\hline Enzyme & Class & CARB & Vaborbactam & Avibactam & Relebactam & QPX7728 \\
\hline KPC-2 & $A$ & + & $110 \pm 30$ & $22 \pm 6$ & $82 \pm 17$ & $2.9 \pm 0.4$ \\
\hline CTX-M-14 & A & - & $110 \pm 40$ & $1.4 \pm 0.4$ & $34 \pm 10$ & $0.94 \pm 0.2$ \\
\hline CTX-M-15 & A & - & $92 \pm 13$ & $0.56 \pm 0.25$ & $76 \pm 3$ & $1.2 \pm 0.1$ \\
\hline SHV-12 & A & - & $56 \pm 11$ & $0.61 \pm 0.19$ & $330 \pm 50$ & $1.9 \pm 0.6$ \\
\hline TEM-10 & A & - & $470 \pm 150$ & $4.3 \pm 1.2$ & $160 \pm 20$ & $2.2 \pm 0.8$ \\
\hline P99 & C & - & $88 \pm 38$ & $26 \pm 7$ & $36 \pm 4$ & $22 \pm 6$ \\
\hline OXA-48 & $\mathrm{D}$ & + & $6.9 \pm 2.3 \times 10^{3}$ & $180 \pm 50$ & $9 \pm 0.3 \times 10^{4}$ & $1.1 \pm 0.4$ \\
\hline OXA-23 & D & + & $1.2 \pm 0.2 \times 10^{5}$ & $3.1 \pm 0.6 \times 10^{3}$ & ND & $1.2 \pm 0.4$ \\
\hline $\mathrm{NDM}-1^{*}$ & $B$ & + & $>1.6 \times 10^{5}$ & $>1.6 \times 10^{5}$ & $>1.6 \times 10^{5}$ & $55 \pm 25$ \\
\hline VIM-1 & $B$ & + & $>1.6 \times 10^{5}$ & $>1.6 \times 10^{5}$ & $>1.6 \times 10^{5}$ & $14 \pm 4$ \\
\hline $\mid \mathrm{MP}-1^{*}$ & $B$ & + & $>1.6 \times 10^{5}$ & $>1.6 \times 10^{5}$ & $>1.6 \times 10^{5}$ & $610 \pm 70$ \\
\hline IMP-26* & B & + & $>1.6 \times 10^{5}$ & $>1.6 \times 10^{5}$ & $>1.6 \times 10^{5}$ & $4.1 \pm 1 \times 10^{3}$ \\
\hline
\end{tabular}

*Imipenem was used as a substrate. Data from Tsivkovski et al. (2020).

as a host to construct a panel of over 55 recombinant strains producing plasmid encoded single $\beta$-lactamases. This panel confirmed the spectrum of $\beta$-lactamase inhibition by QPX7728 observed in biochemical assays and expanded the list of enzymes inhibited by QPX7728 (Table 2). For purposes of comparative characterization with different $\beta$-lactamase inhibitors, substrates such as ceftazidime and piperacillin were used due to their combined lability to $\beta$-lactamases from all classes.

Based on results of both biochemical and microbiological assays, QPX7728 is a potent inhibitor of Class A ESBLs such as CTX-M, SHV, TEM, VEB, and PER, including some variants such as PER-4 that are less susceptible to inhibition with avibactam. It also inhibits Class A carbapenemases identified in various species of gram-negative bacteria such as KPC, SME, NMCA, GES-20, VCC-1, BKC-1. Its spectrum of inhibition includes many Class C $\beta$-lactamases, both plasmidic, such as SMY and MIR from Enterobacterales, and chromosomal enzymes from Enterobacterales, $P$. aeruginosa and A. baumannii. QPX7728 appears to be equally potent in inhibiting diverse Class $\mathrm{D} \beta$ lactamases, including carbapenemases such as OXA-48 from Enterobacterales and OXA-23/24/72/58 from A. baumannii.

Importantly, QPX7728 inhibits many metallo- $\beta$-lactamases from the B1 (Galleni et al., 2001) sub-class of the Class B family including NDM, VIM, CcrA1, IMP, and GIM but not SPM-1. The B1 sub-class represents the most common MBL enzymes; there are two other classes of MBLs, B2 and B3, consisting of less common $\beta$-lactamases. There are significant structural and mechanistic differences between B1 vs. B2 and particularly, B3 MBLs (Galleni et al., 2001). B2 and B3 MBLs such as L1 from Stenotrophomonas maltophilia are not or poorly inhibited by QPX7728.

\section{QPX7728 Inhibits Serine and Metallo Enzymes With a Significantly Different Kinetics}

Detailed kinetic studies performed with QPX7728 and described in Tsivkovski et al. (2020) indicated that inhibition of all the tested serine enzymes by QPX7728 was consistent with slow tightbinding mechanism associated with progressive inactivation. QPX7728 acts by a two-step inhibition mechanism in which a non-covalent complex is formed first and subsequently proceeds to a covalent interaction. It is expected that the boron atom of QPX7728 forms covalent bond with the catalytic serine residue of the $\beta$-lactamase. Such covalent complexes were noted for other boronic BLIs (Rojas et al., 2016). Based on kinetic analysis, QPX7728 is a highly efficient inhibitor of serine $\beta$-lactamases with $\mathrm{K}_{\text {on }} \mathrm{s}$ in a $10^{4} \mathrm{M}^{-1} \mathrm{~s}^{-1} \times 10^{6} \mathrm{M}^{-1} \mathrm{~s}^{-1}$ range (Table 3). In the case of KPC-2 inhibition, QPX7728 appears to be almost 100-fold more efficient than vaborbactam $\left[\mathrm{k}_{2} / \mathrm{K}\right.$ of $5.5 \times 10^{3}$ (Tsivkovski and Lomovskaya, 2019)] and ca. 10-fold more efficient than avibactam $\left[\mathrm{k}_{2} / \mathrm{K}\right.$ of $1.3 \times 10^{4}$ (Ehmann et al., 2013) $]$ and relebactam $\left[\mathrm{k}_{2} / \mathrm{K}\right.$ of $2.5 \times 10^{4}$ (Papp-Wallace et al., 2018)].

QPX7728 inhibition of serine $\beta$-lactamases was found to be reversible in all the studied enzymes; however, the stability of the QPX7728- $\beta$-lactamase complexes differed depending on the enzyme. The longest target residence time, $\sim 3.5 \mathrm{~h}$, was detected for CTX-M-15 and the shortest, 5-20 min, for OXA carbapenemases from A. baumannii (Table 3 ). The residence 
TABLE 2 | In vitro potency (MIC, $\mu \mathrm{g} / \mathrm{ml}$ ) of ceftazidime and piperacillin alone and in combination with BLIs against the panel of engineered strains producing single $\beta$-lactamases.

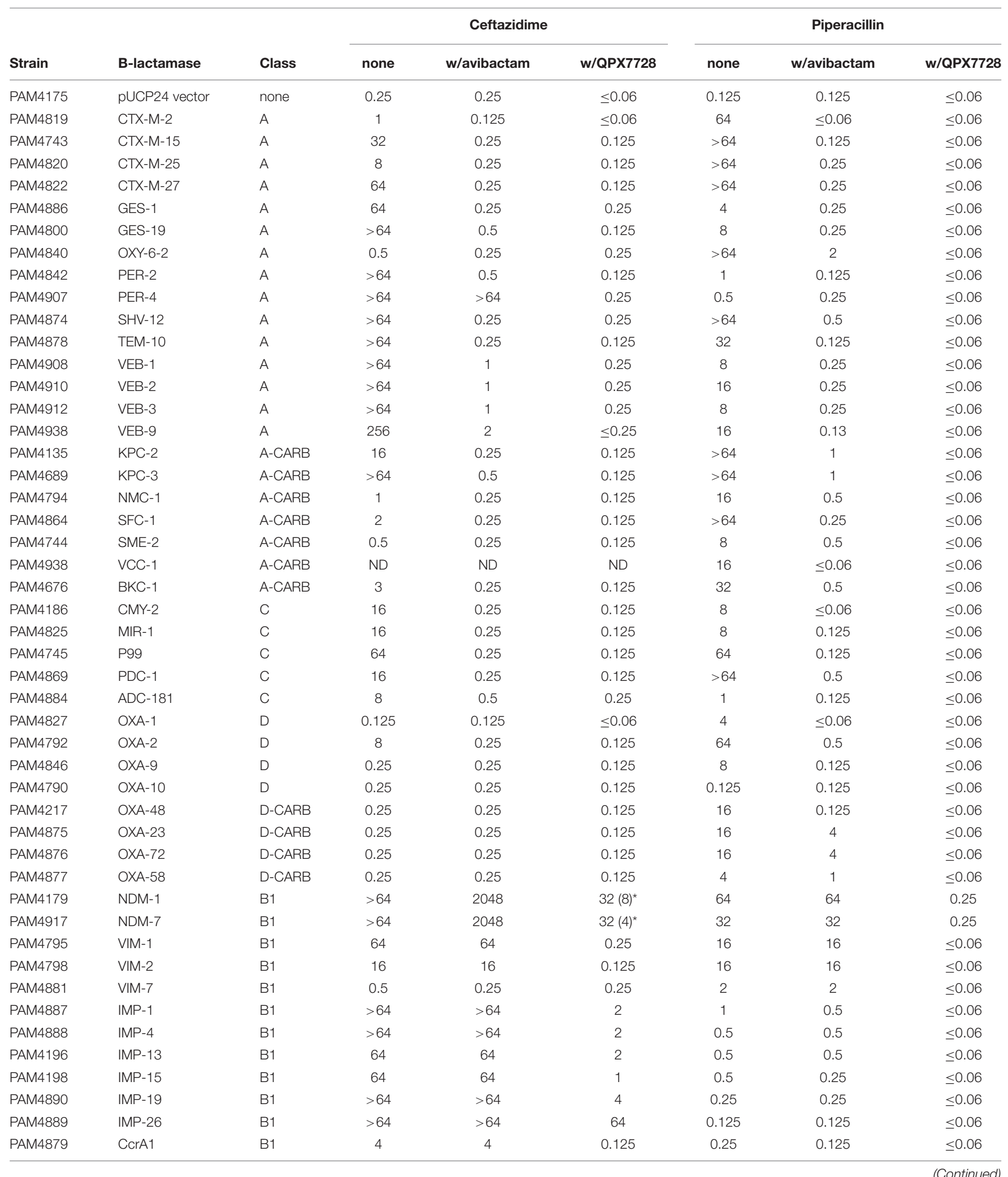


TABLE 2 | Continued

\begin{tabular}{|c|c|c|c|c|c|c|c|c|}
\hline \multirow[b]{2}{*}{ Strain } & \multirow[b]{2}{*}{ B-lactamase } & \multirow[b]{2}{*}{ Class } & \multicolumn{3}{|c|}{ Ceftazidime } & \multicolumn{3}{|c|}{ Piperacillin } \\
\hline & & & none & w/avibactam & w/QPX7728 & none & w/avibactam & w/QPX7728 \\
\hline PAM4883 & GIM-1 & B1 & $>64$ & 64 & 0.5 & $>64$ & $>64$ & 2 \\
\hline PAM4885 & SPM-1 & B1 & $>64$ & $>64$ & $>64$ & 32 & 32 & 8 \\
\hline PAM4880 & L1 & B3 & 64 & 64 & 64 & 32 & 32 & 8 \\
\hline
\end{tabular}

Genes encoding various $\beta$-lactamases were cloned in the plasmid pUCP24 and introduced in the strain of P. aeruginosa that lacks major efflux pumps

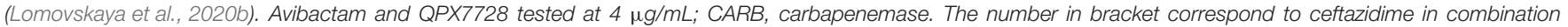
with QPX7728 at $8 \mu \mathrm{g} / \mathrm{ml}$ Data from Lomovskaya et al. (2020b).

TABLE 3 | Kinetic parameters of $\beta$-lactamase inhibition by QPX7728.

\begin{tabular}{|c|c|c|c|c|c|}
\hline Enzyme & $k_{2} / K\left(M^{-1} s^{-1}\right)$ & $k_{o f f}, s^{-1}$ & Residence time, $\min$ & $\mathrm{K}_{d}, \mathrm{nM}$ & Stoichiometry \\
\hline KPC-2 & $3.6 \pm 0.1 \times 10^{5}$ & $9.0 \pm 1.4 \times 10^{-5}$ & $189 \pm 31$ & $0.25 \pm 0.03$ & 1 \\
\hline KPC-3 & $4.1 \pm 1.0 \times 10^{5}$ & $1.26 \pm 0.07 \times 10^{-4}$ & $133 \pm 8$ & $0.31 \pm 0.06$ & 1 \\
\hline BKC-1 & $1.82 \pm 0.04 \times 10^{6}$ & $1.1 \pm 0.1 \times 10^{-4}$ & $154 \pm 14$ & $0.060 \pm 0.005$ & 1 \\
\hline $\mathrm{FRI}-1$ & $1.15 \pm 0.03 \times 10^{6}$ & $1.2 \pm 0.2 \times 10^{-4}$ & $138 \pm 24$ & $0.11 \pm 0.01$ & 1 \\
\hline SME-2 & $1.2 \pm 0.1 \times 10^{6}$ & $1.8 \pm 0.2 \times 10^{-4}$ & $94 \pm 11$ & $0.15 \pm 0.02$ & 1 \\
\hline CTX-M-15 & $6.9 \pm 0.6 \times 10^{5}$ & $8.0 \pm 1.0 \times 10^{-5}$ & $220 \pm 33$ & $0.11 \pm 0.02$ & 1 \\
\hline SHV-12 & $1.1 \pm 0.2 \times 10^{5}$ & $3.0 \pm 0.2 \times 10^{-3}$ & $5.5 \pm 0.3$ & $28 \pm 4$ & 1 \\
\hline TEM-43 & $1.9 \pm 0.3 \times 10^{6}$ & $3.2 \pm 0.2 \times 10^{-4}$ & $53 \pm 3$ & $0.17 \pm 0.02$ & 1 \\
\hline P99 & $6.3 \pm 0.7 \times 10^{4}$ & $3.3 \pm 0.3 \times 10^{-5}$ & $506 \pm 51$ & $0.53 \pm 0.06$ & 1 \\
\hline OXA-48 & $2.75 \pm 0.09 \times 10^{6}$ & $3.6 \pm 0.2 \times 10^{-4}$ & $47 \pm 3$ & $0.13 \pm 0.01$ & 1 \\
\hline OXA-23 & $9.9 \pm 0.6 \times 10^{5}$ & $1.6 \pm 0.2 \times 10^{-3}$ & $11 \pm 2$ & $1.6 \pm 0.2$ & 2 \\
\hline OXA-24 & $1.5 \pm 0.2 \times 10^{6}$ & $9.0 \pm 1.0 \times 10^{-4}$ & $20 \pm 3$ & $0.58 \pm 0.10$ & 1 \\
\hline OXA-58 & $1.07 \pm 0.08 \times 10^{6}$ & $3.5 \pm 0.3 \times 10^{-4}$ & $4.8 \pm 0.5$ & $3.2 \pm 0.3$ & 1 \\
\hline NDM-1 & ND & ND & ND & $32 \pm 14$ & ND \\
\hline VIM-1 & ND & ND & ND & $7.5 \pm 2.1$ & ND \\
\hline IMP-1 & ND & ND & ND & $240 \pm 30$ & ND \\
\hline
\end{tabular}

ND, not determined. Data from Tsivkovski et al. (2020).

time of QPX7728 for KPC-2, 3 h, was slightly longer than that of avibactam ( $\sim 2 \mathrm{~h})$ (Ehmann et al., 2013), and relebactam (1.5 h) (Papp-Wallace et al., 2018) but shorter than that for vaborbactam ( 6 h) (Tsivkovski and Lomovskaya, 2019). Despite the 2-3-fold lower $k_{\text {off }}$ values of vaborbactam inhibition of KPC-2 and KPC3, QPX7728 $\mathrm{K}_{d}$ values for these enzymes were 10 to 50 -fold lower than vaborbactam owing to its ca. 100-fold higher inactivation efficiency of KPCs (Tsivkovski and Lomovskaya, 2019; Table 3).

While QPX7728 behaves as a two-step covalent slow off-rate inhibitor of serine enzymes, its inhibition of metallo can be described by a classical Michaelis-Menten kinetics with a simple one-step complex formation followed by rapid dissociation of the enzyme-inhibitor complex. It is a competitive inhibitor with no sign of progressive inactivation and with $\mathrm{K}_{i}$ s ranging from $\sim 7 \mathrm{nM}, \sim 32 \mathrm{nM}$ and $\sim 240 \mathrm{nM}$ for VIM-1, NDM-1 and IMP-1, respectively (Table 3 ).

\section{Structural Studies Are Consistent With the Mechanism of QPX7728 Inhibition and Provide Insights Into Its Potency, Breadth of Spectrum, and Its Limitations}

The structures of KPC-2, NDM-1, VIM-2 and OXA-48 complexed with QPX7728 were solved at high resolution ranging from 1.05 to $1.85 \AA$ (Hecker et al., 2020). Structures of QPX7728 complexed to KPC-2, and OXA-48 provide clear evidence of the covalent bond between the boron atom of the inhibitor and the catalytic serine residue of the enzymes. In the metallo enzymes, the covalent bond is observed between the boron atom and the catalytic water molecule present in the active site of NDM-1 and VIM-2. Of note, the boron becomes tetrahedral on binding to both MBLs and SBLs (Figure 2).

Superimposition of $\beta$-lactamases using the ligand shows that QPX7728 binds all of them in a nearly identical conformation. The most significant difference appears to be in the orientation of carboxylate in metallo- vs. non metallo enzymes; it is closer to coplanar in MBLs and closer to orthogonal in others (Figure 3). All enzymes engage the carboxylate in multiple strong interactions, but details can vary and there may be hydrogen bonds to side chain (Thr)hydroxyl, backbone amide or a water molecule as well as interactions with cationic side chains (Lys or Arg) or metal ion $(\mathrm{Zn})$ (Figure 3B). Common to all $\beta$-lactamase enzymes are lipophilic interactions of the 'back' edge of the ring system, which is always packed against lipophilic, predominantly aromatic, sidechains which otherwise vary significantly from enzyme to enzyme (Figure 3A).

Recently the structure of the KPC-vaborbactam complex was published by two groups (Pemberton et al., 2020; 

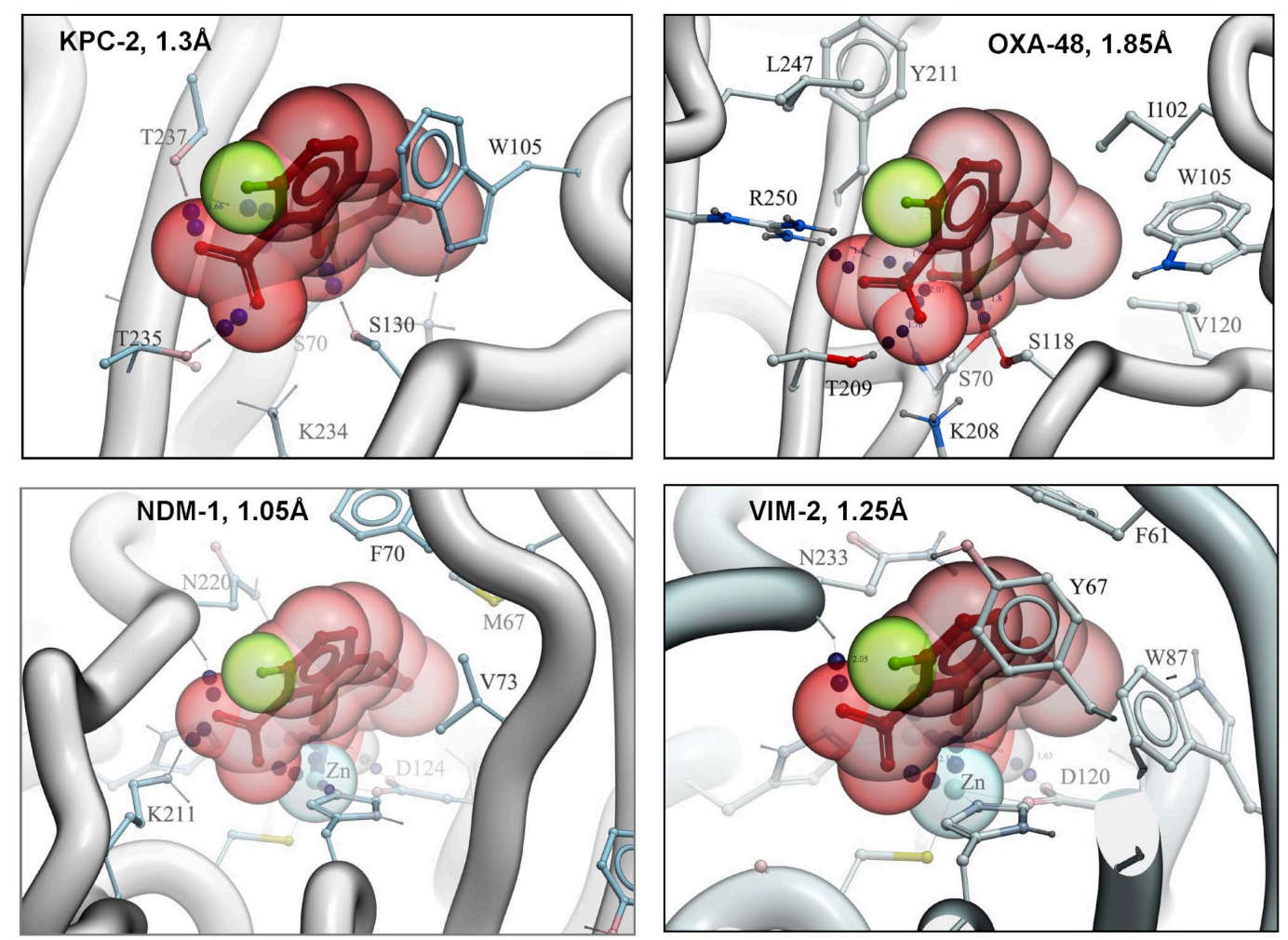

FIGURE 2 | High resolution structures of QPX7728 with KPC-2, OXA-48, NDM-1, and VIM-2. Accession codes are the following: 6V1J (KPC-2: QPX7728), 6V1M (NDM-1: QPX7728), 6V1O (OXA-48: QPX7728), and 6V1P (VIM-2: QPX7728). Modified from Hecker et al. (2020).
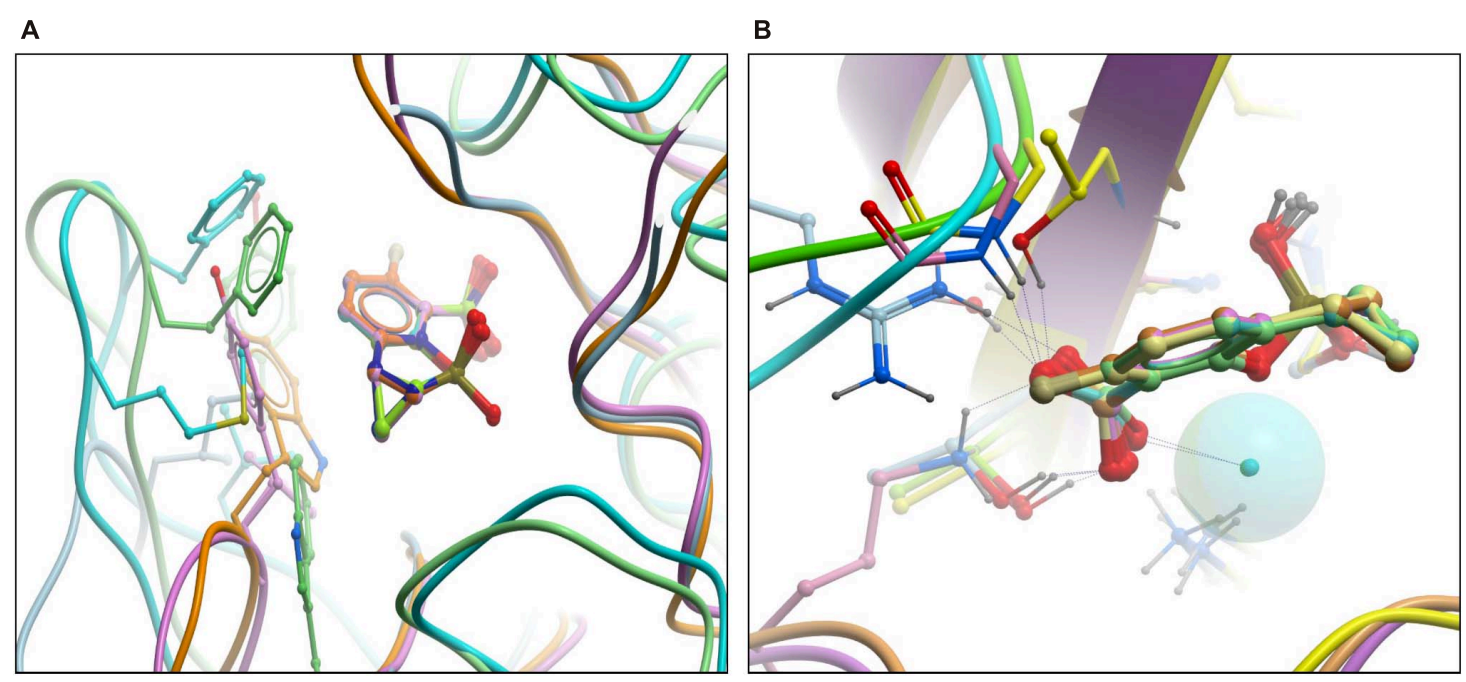

FIGURE 3 | High resolution structures of RPX7728 with multiple enzymes: superimposition of $\beta$-lactamases using the ligand. (A) The aromatic/lipophilic interactions are shown. (B) Close-up of carboxylate interactions and orientation.

Tooke et al., 2020) and there are both similarities and differences with KPC-QPX7728 complex (Figure 4A). Both boronates are covalently attached to S70 and the positioning of carboxylate is nearly identical but the boronate moieties are positioned somewhat differently. Vaborbactam inserts both exocyclic and endocyclic oxygen atoms into oxyanion hole, while only exocylic oxygen of QPX7728 is in the oxyanion hole. Endocyclic oxygen is coordinated by the S130 side chain. Overall QPX7728/KPC interactions are focused on the core conserved active site which QPX7728 fully occupies via the double ring system, forming more 
extensive lipophilic interactions with aromatic W105 side chain and other lipophilic surfaces. In contrast, vaborbactam picks up more interactions on the periphery, including a hydrogen bond to N132 and lipophilic contacts of the thiophene 'sidechain.'

As discussed in Tsivkovski et al. (2020), a notable feature of QPX7728 molecular structure is its limited flexibility - the system of three rings constrains almost the entire molecule, and the only remaining free torsion (carboxylate rotation) is restricted by conjugation. Near-total rigidity of QPX7728 should result in a negligible conformational entropy loss upon binding, and likely contributes to its enhanced potency as compared to vaborbactam, which has 5 rotatable bonds. As evidenced by X-ray structures (Hecker et al., 2015), the vaborbactam ring also can adopt two alternative low energy conformations, for a total of 6 degrees of freedom. Assuming entropic loss of $\mathrm{T} \Delta \mathrm{S} \sim 0.6 \mathrm{kcal} / \mathrm{mol}$ per degree of freedom, the vaborbactam molecule might experience a penalty in binding free energy $\Delta \mathrm{G}$ of as much as $3.6 \mathrm{kcal} / \mathrm{mol}$, corresponding to a several hundred-fold difference in $\mathrm{K}_{d}$.

As suggested in the same study (Tsivkovski et al., 2020), one factor that likely contributes to the exceptional breadth of inhibition spectrum of QPX7728 is that its structure is very compact. The entire inhibitor molecule is only slightly larger than the $\beta$-lactam substrate core 5/4 ring system, and it fits within the well-conserved immediate vicinity of the catalytic site. Lack of any peripheral moieties in contact with more distal (and variable) regions of the enzyme may allow the inhibitor to avoid unfavorable interactions that sequence/structure variations could otherwise introduce (Figure 4B).

The crystal structure of QPX7728/NDM-1 complex (Hecker et al., 2020) revealed the key interactions that contribute to the high-affinity binding of the inhibitor to MBLs. Three distinct features can be discerned: (1) the ligand is coordinating both zinc ions at the core of the active site via one of the carboxylic acid's oxygen atoms, the boronate ester oxygen, and its hydroxyl; (2) the ligand's phenyl and cyclopropyl rings form extensive lipophilic contacts, largely with the sidechains in the loop L65:V73 that caps the active site; and (3) the ligand's carboxylate forms a salt bridge and/or charge assisted hydrogen bonds with a positively charged sidechain/other hydrogen bond donors of the enzyme (Figure 5A).

As described in Lomovskaya et al. (2020b), we modeled the putative QPX7728/L1 complex by docking and attempted to identify the structural features that may preclude QPX7728 activity against $\beta$-lactamase L1. Docking simulation resulted in a bound pose of the ligand that retained coordination of the two metal ions in a manner closely resembling available experimental structures of complexes with other MBLs (Figure 5B). However, L1 structure lacks a positively charged sidechain analogous to K211 of NDM-1, therefore in the putative L1 complex QPX7728 did not form a salt bridge to the enzyme. Furthermore, QPX7728 hydrophobic interactions with L1 were limited due to the lack of the 'capping' loop in L1, where this loop is truncated to a short hairpin. Thus, it appears plausible that loss of lipophilic interactions with the 'capping' loop and the absence of a salt bridge to K211 (or similar residue), drastically reduce QPX7728 affinity to L1, in comparison to its affinity to most other MBLs. These observations suggest that significant modifications of
QPX7728 chemical structure may be necessary to achieve potent inhibition of this sub-family of MBLs.

In conclusion, QPX7728 is a potent inhibitor of numerous $\beta$ lactamases from all molecular classes. Its $\beta$-lactamase inhibition profile is significantly enhanced compared to the recently approved BLIs. In addition to serine Class A, Class C and some Class D broad-spectrum $\beta$-lactamases and carbapenemases, the activity of QPX7728 includes serine Class D carbapenemases that are widely distributed in carbapenem-resistant $A$. baumannii (OXA-23/OXA-40) as well as metallo $\beta$-lactamases belonging to the B1 sub-class (NDM, VIM, IMP).

\section{QPX7728 Is Significantly Less Affected by General Intrinsic Resistance Mechanisms in Gram-Negative Bacteria}

Gram-negative bacteria are inherently resistant to a variety of antibiotics (Nikaido, 2003; Li et al., 2015). This permanent multi-drug resistant state is due to a combined effect of the low permeability outer membrane and the activity of efflux transporters capable of recognizing and extruding multiple unrelated substrates, the so-called multi-drug resistance efflux pumps. B-lactams are one of the few classes of antibiotics that do have representatives with clinically useful activity against these multi-drug resistant gram-negative organisms; however, their activity can be decreased by permeability/efflux-mediated intrinsic mechanisms. B-lactamase inhibitors cannot overcome this type of resistance and thus their inhibitory potency might be decreased by these same intrinsic mechanisms (Shields et al., 2015; Nelson et al., 2017; Gomez-Simmonds et al., 2018; Wilson et al., 2019). Hence, the important objective of our BLI lead optimization program was to minimize the impact of these intrinsic resistance mechanisms on BLI potency.

\section{Impact of Porin Mutations and Increased Efflux in K. pneumoniae on QPX7728 Potency}

In Enterobacterales, major outer membrane porins OmpF/OmpK35 and OmpC/OmpK36 are important entry routes for $\beta$-lactams and $\beta$-lactamase inhibitors (Pages et al., 2008). Consequently, porin mutations affect the potency of many $\beta$-lactams and BL/BLI combinations (Lomovskaya et al., 2017). The pump which is the most relevant for $\beta$-lactam efflux is AcrAB (Pages et al., 2009). Using a set of KPC-producing porin/efflux mutants of $K$. pneumoniae, we demonstrated that combined effect of inactivation of the major porins OmpK35 and OmpK36 and increased efflux activity results in ca. 32-fold reduction of the potency of QPX7728 to completely inhibit KPC; the potency was reduced from 0.125 to $4 \mu \mathrm{g} / \mathrm{ml}$ (Lomovskaya et al., 2020a). This contrasts with a significantly stronger 256-fold reduction of the potency of vaborbactam by the same mutations (from 0.25 to $64 \mu \mathrm{g} / \mathrm{ml}$ ) (Lomovskaya et al., 2017).

\section{Impact of Efflux and OprD in Pseudomonas aeruginosa and Efflux in Acinetobacter baumannii on QPX7728 Potency}

The outer membrane of $P$. aeruginosa lacks permanently open porins that are present in Enterobacterales (Chevalier et al., 2017) which significantly enhances its potential as permeability 
A

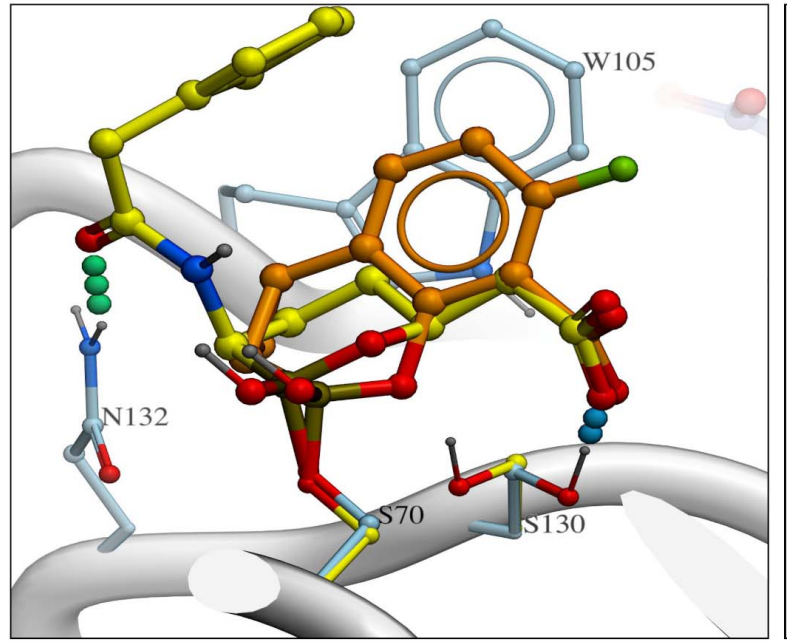

B

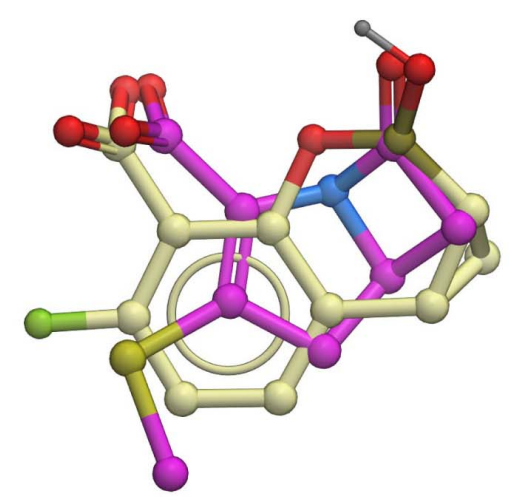

FIGURE 4 | (A) Comparison of QPX7728 and vaborbactam in complex with KPC-2 with carbon atoms of QPX7728 and vaborbactam in light brown and in yellow carbon atoms, respectively [accession codes are the following: 6V1J (KPC-2:QPX7728); 6TD0 (KPC-2:vaborbactam)]. (B) Superposition of QPX7728 (crème carbon atoms) and the core ring system of penem antibiotics (magenta carbon atoms).

A

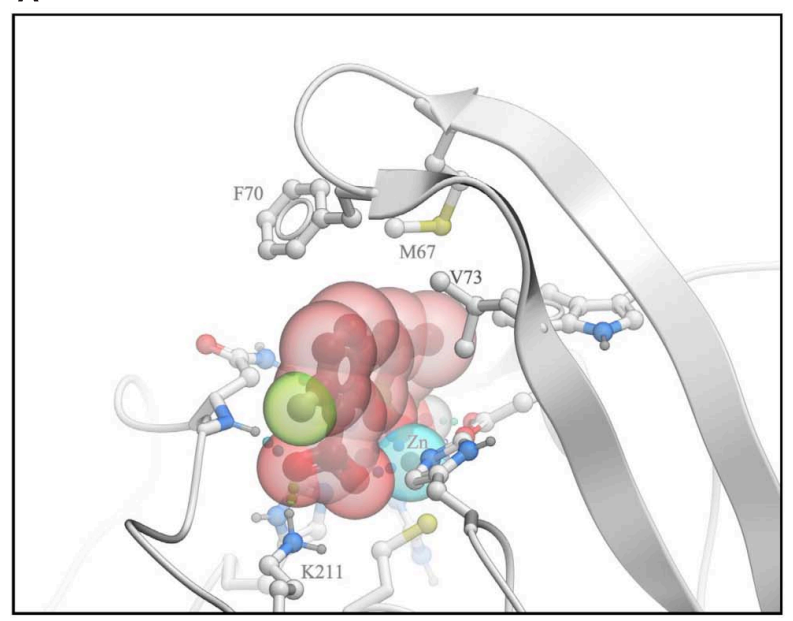

B

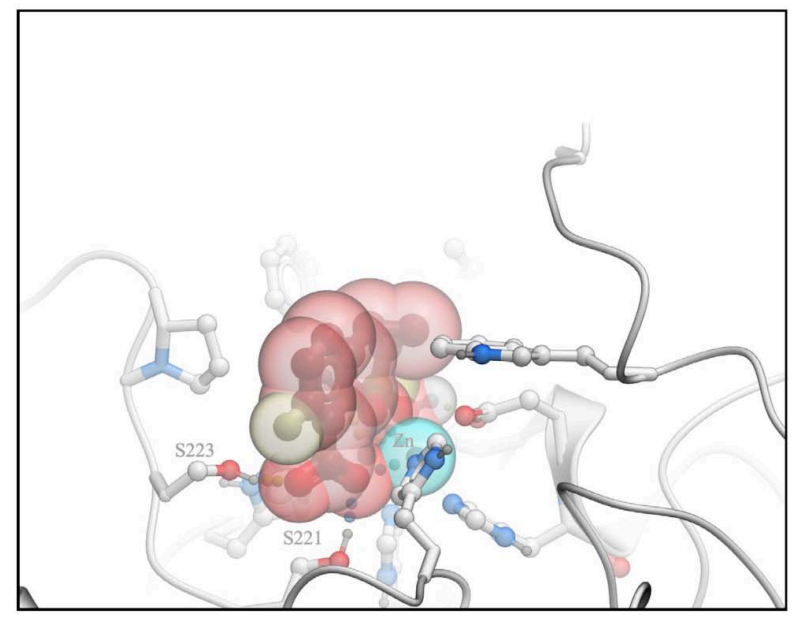

FIGURE 5 | Analysis of interactions of QPX7728 with NDM-1 and L1 B-Lactamases. (A) The crystal structure of QPX7728/NDM-1 complex. (B) Model of the putative QPX7728/L1 complex. Modified from Lomovskaya et al. (2020b).

barrier. It also possesses multiple efflux pumps; working together these mechanisms provide the powerful protection against antipseudomonal toxins that include clinically useful antibiotics (Lister et al., 2009; El Zowalaty et al., 2015; Sommer et al., 2020). The MexAB-OprM efflux pump is particularly relevant for resistance to many $\beta$-lactam antibiotics (Srikumar et al., 1999), BLIs (Li et al., 1998), and BL/BLI combinations (Chalhoub et al., 2018; Castanheira et al., 2019). The basic amino acid porin OprD is used as a gate of entry of carbapenems (Ochs et al., 1999) and oprD mutants have an increased carbapenem resistance (Lister et al., 2009). Multiple multidrug resistance efflux pumps are described in A. baumannii: among them AdeABC and AdeIJK are particularly important for antibiotic resistance and general defense (Coyne et al., 2011).

In a panel of isogenic strains of $P$. aeruginosa that produced KPC (as a reporter) in the background of efflux/oprD mutations, we demonstrated that these mutations had a minimal effect on the BLI activity of QPX7728 (Lomovskaya et al., 2020a). No more than two-fold reduction in potency was observed when the mexAB-oprM efflux operon was overexpressed. For comparison, the potency of vaborbactam was reduced 8-fold or more due to overproduction of the MexAB-OprM or the MexEF-OprN 
efflux pumps, respectively (Lomovskaya et al., 2020a; Table 4). Inactivation of the carbapenem porin $\mathrm{OprD}$ did not have any effect on QPX7728 potency (Lomovskaya et al., 2020a).

The experiments using isogenic panel of Acinetobacter baumannii mutants producing OXA-23 in the background of overexpression of efflux pumps also showed no effect of efflux on the potency of QPX7728 to inhibit $\beta$-lactamase (Lomovskaya et al., 2020a).

In conclusion, while mutations in major porins of Enterobacterales decrease the potency of QPX7728, it still maintains excellent inhibitory activity in strains with multiple porin mutations. Overexpression of efflux pumps in various target organisms (Enterobacterales, Pseudomonas, Acinetobacter) does not reduce the antibiotic potentiation activity of QPX7728.

\section{QPX7728 in Combination With Various $\beta$-Lactams Against Clinical Isolates of Target Pathogens}

Several studies were performed to determine the potency of QPX7728 in combination with various $\beta$-lactams against large panels of clinical isolates of target pathogens. We used a newly developed metric called target potentiation concentration or TPC [described in detail in Nelson et al. (2020a)] to separately determine the whole-cell BLI potency of QPX7728. TPC is the concentration of QPX7728 that is required to reduce antibiotic MIC for a given strain to or below the existing susceptibility breakpoint of the partner antibiotic tested. Conceptually, it is the MIC of the BLI determined in the presence of a partner antibiotic present at a concentration that corresponds to its susceptibility breakpoint. By assessing the distribution of TPCs in the population of strains, one can determine $\mathrm{TPC}_{90}$, or the concentration of QPX7728 that shifts $90 \%$ of the tested isolates at or below susceptibility breakpoint for the partner antibiotic. This metric is extremely useful when one wants to compare the potency of a potentiating agent in combination with different antibiotics against different panels of target organisms and to evaluate the impact of various genotypes and phenotypes on its potency.

In addition, $\mathrm{TPC}_{90}$ is an important metric that will be used to link in vitro BLI potency to exposures required for BLI activity in vivo. Once safe and efficacious BLI exposures established based on $\mathrm{TPC}_{90}$ and various PK-PD studies are achieved, $\mathrm{TPC}_{90}$ will be also important to select concentration of QPX7728 for in vitro susceptibility testing.

\section{QPX7728 Restores the Potency of Various $\beta$-Lactam Antibiotics Against Enterobacterales With Diverse Acquired and Intrinsic Mechanisms of $\beta$-Lactam Resistance}

In the study by Nelson et al. (2020a), increasing concentrations of QPX7728 (1-16 $\mu \mathrm{g} / \mathrm{ml})$ were shown to significantly enhance the potency of meropenem against a panel of 598 molecularly characterized carbapenem-resistant strains of Enterobacterales
(CRE), consisting of serine and metallo $\beta$-lactamases producers as well as non-carbapenemase producing CREs (Figure 6A). This study determined the TPC 90 of QPX7728 for this panel of strains using meropenem susceptibility breakpoint of $8 \mu \mathrm{g} / \mathrm{ml}$ [meropenem PK-PD breakpoint at a dose of $2 \mathrm{~g}$ administered every $8 \mathrm{~h}$ by 3 -h infusion (Kuti et al., 2003; Lee et al., 2010)]. The $\mathrm{TPC}_{90}$ of QPX7728 for studied CRE isolates was $4 \mu \mathrm{g} / \mathrm{ml}$.

Importantly, the potency of meropenem was increased by QPX7728 against all groups of carbapenemase producing CREs, consisting of KPC-, OXA-48-like- and MBL-producing strains, as well as against non-carbapenemse-producing CRE strains. $\mathrm{MIC}_{90}$ of meropenem was $1 \mu \mathrm{g} / \mathrm{mL}$ or less when QPX7728 was tested at $8 \mu \mathrm{g} / \mathrm{ml}$; at this concentration of QPX7728 (as well as at $4 \mu \mathrm{g} / \mathrm{ml}$ ) meropenem/QPX7728 was the most potent BL/BLI combination tested against all groups of CRE (Figure 7).

As expected from the studies of laboratory mutants (Lomovskaya et al., 2020a), porin mutations reduced the potency of meropenem/QPX7728. However, when meropenem was combined with QPX7728 at 4 or $8 \mu \mathrm{g} / \mathrm{ml}$, more than $90 \%$ of both MBL-negative and MBL-positive CRE with various defects in OmpK36/OmpC were susceptible using a meropenem breakpoint of $8 \mu \mathrm{g} / \mathrm{ml}$ (Table 5); thus, the QPX7728 TPC $_{90}$ values were still in the $4-8 \mu \mathrm{g} / \mathrm{ml}$ range even for the subsets of strains that had a combination of carbapenemase production and non- $\beta$-lactamase-mediated resistance mechanisms.

We also assessed enhancement of the potency of cefepime and aztreonam by QPX7728 (at concentrations from 1 to $16 \mu \mathrm{g} / \mathrm{ml}$ ) against the panel of 756 of ESBL and CRE producing strains of Enterobacterales with the objective to determine the $\mathrm{TPC}_{90}$ of QPX7728 in combination with these antibiotics.

QPX7728 significantly increased potency of both antibiotics against this challenge panel: $\mathrm{MIC}_{90}$ for both antibiotics was decreased from $>64$ to $2 \mu \mathrm{g} / \mathrm{ml}$ and $0.25 \mu \mathrm{g} / \mathrm{ml}$ with QPX7728 at 4 and $8 \mu \mathrm{g} / \mathrm{ml}$, respectively (Figures 6B,C). The QPX7728 TPC $_{90}$, determined at $8 \mu \mathrm{g} / \mathrm{ml}$ of both antibiotics (FDA susceptible breakpoint), was $4 \mu \mathrm{g} / \mathrm{ml}$ in combination with either cefepime or aztreonam.

Overall, combinations of QPX7728 with multiple antibiotics were highly potent against the majority of strains, including those which had various carbapenemases produced in the background of mutations resulting in decreased permeability. The potency of QPX7728 as measured by TPC 90 was the same, irrespective of the antibiotic combined with QPX7728.

\section{QPX7728 in Combination With Several $\beta$-Lactams Has Excellent Potency Against KPC Mutants That Are Resistant to Ceftazidime-Avibactam}

Mutations in KPC genes that confer resistance to ceftazidimeavibactam were first selected in laboratory conditions (Livermore et al., 2015), but soon were identified in isolates recovered from patients treated with this combination (Shields et al., 2017). Based on several studied cases, this resistance appears to be due to both an increase in the efficiency of ceftazidime hydrolysis by KPC and reduced affinity of the avibactam/KPC interaction (Barnes et al., 2017; Tsivkovski and Lomovskaya, 2020). Using a 
TABLE 4 | Evaluation of the impact of various efflux mutations on potency of QPX7728 (PV 50$)$ to enhance antibiotic activity.

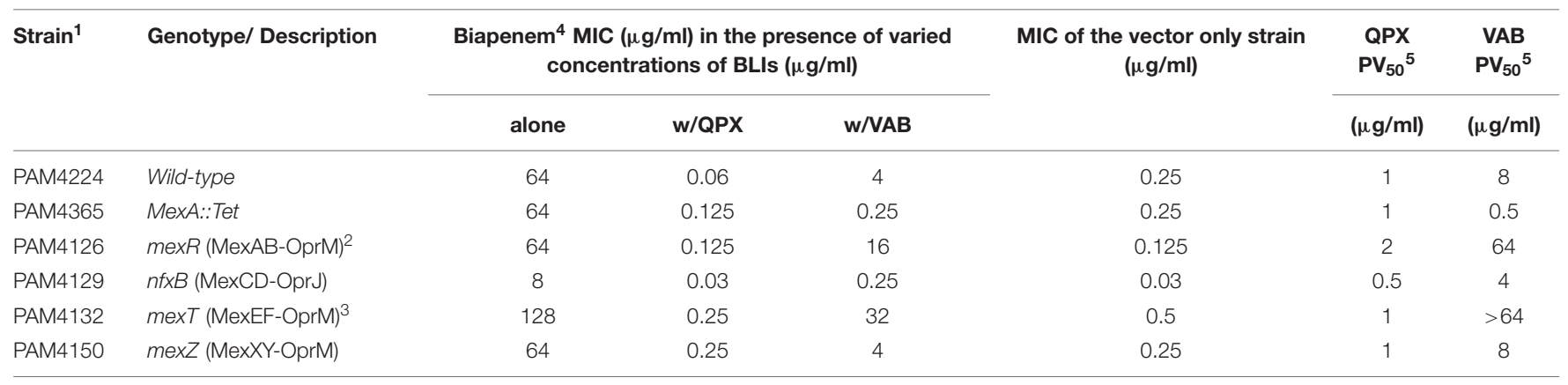

${ }^{1} \mathrm{KPC}-2$ was introduced into isogenic strains of $P$. aeruginosa containing various efflux mutations and used as a reporter for the $Q P X 7728$ BLI activity. ${ }^{2}$ Mutations in regulatory genes result in overproduction of specific efflux complexes that are shown in brackets. ${ }^{3}$ The production of a carbapenem porin OprD is reduced by the same mutation in mexT that leads to overproduction of the MexEF-OprN efflux pump. ${ }^{4}$ Biapenem was chosen as a reporter antibiotic because it's activity against P. aeruginosa is not affected by efflux. The effect of increasing concentrations of QPX7728 on biapenem MIC against the strains producing KPC-2 in the background of various efflux mutations was determined in a standard checkerboard experiment. ${ }^{5} \mathrm{BLI}$ potency was defined as $P V_{50}$ (PV stands for potentiation value) which is a concentration of a BLI required to achieve $50 \%$ of antibiotic potentiation effect (conceptually equivalent to $E C_{50}$ ). It is determined based on the concentration-response curve where antibiotic MICs are plotted vs. BLI concentrations. Data from Lomovskaya et al. (2020a).

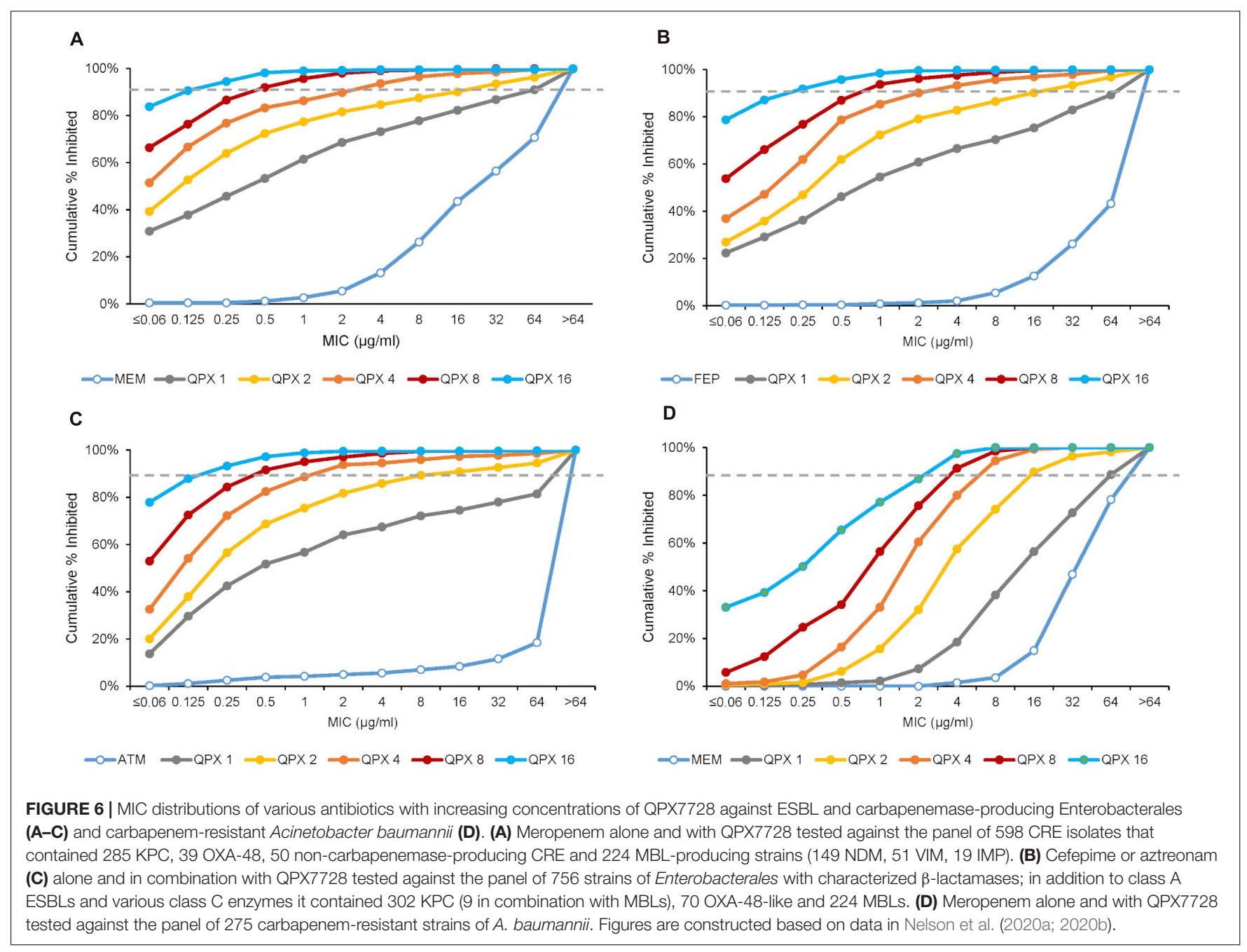

set of single-step ceftazidime-avibactam resistant mutants with various mutations in the bla $a_{K P C}$ selected from a diverse KPCproducing strains of Klebsiella pneumoniae, we demonstrated that these mutations had minimal effect on the MIC of QPX7728 in combination with ceftazidime, cefepime or meropenem (Nelson et al., 2019). Depending on the strain or specific 

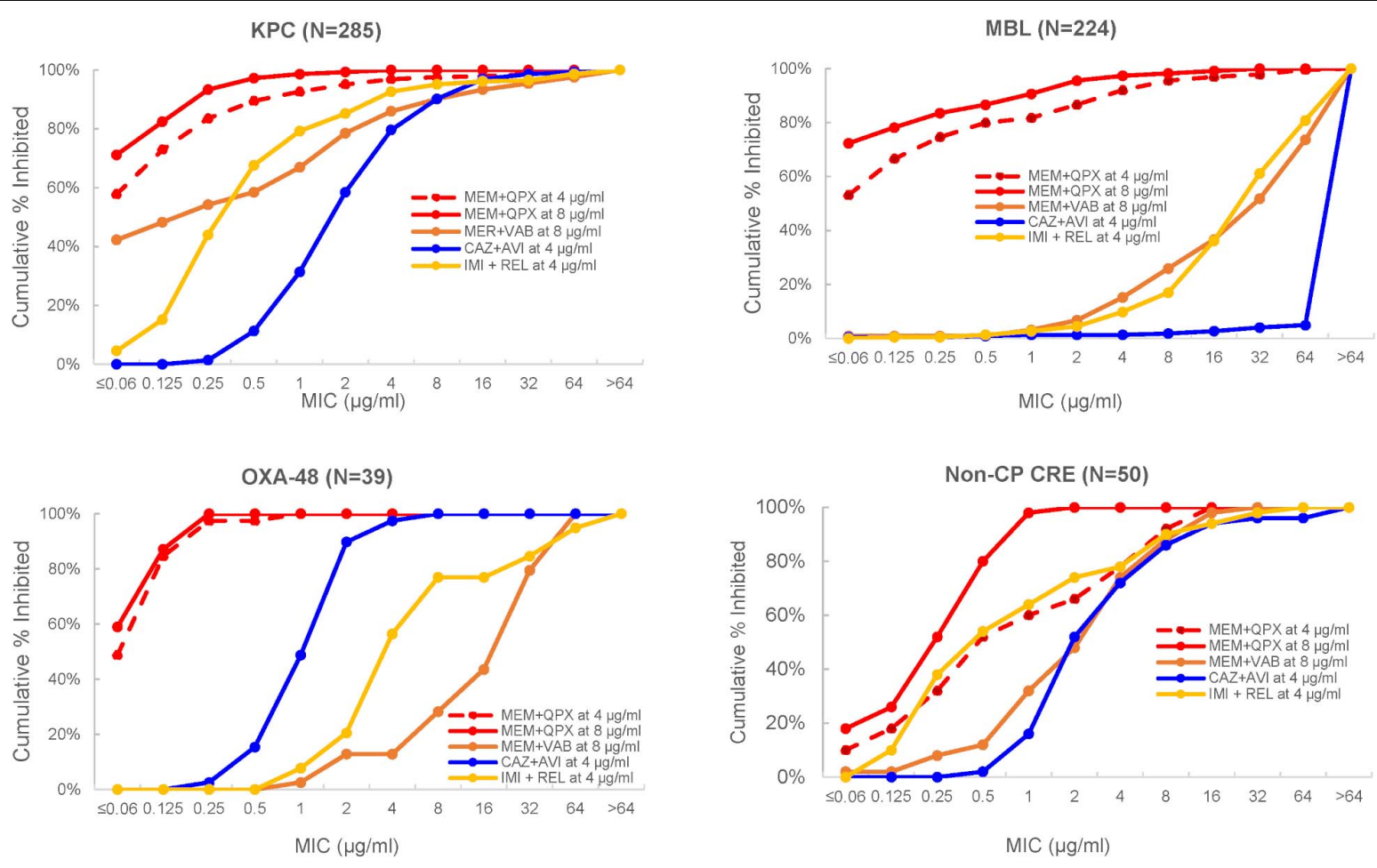

FIGURE 7 | MIC distributions of various $\beta$-lactam/BLI combinations against carbapenem-resistant Enterobacterales according to the type of CRE. MEM, meropenem; CAZ, ceftazidime; IMI, imipenem; AVI, avibactam; QPX, QPX7728; REL, relebactam; Non-CP CRE, non-carbapenemase producing CRE. Adapted with modifications from Nelson et al. (2020a).

mutation, ceftazidime-avibactam MIC values were raised from 4 to $>64$-fold, resulting in MIC values ranging from 16 to $>128 \mu \mathrm{g} / \mathrm{ml}$ (Table 6). The majority of mutants had no increase in ceftazidime-QPX7728, meropenem-QPX7728 or cefepimeQPX7728 MIC values. Meropenem-QPX7728 and cefepimeQPX7728 MIC values of the mutants ranged from $\leq 0.125$ to $0.25 \mu \mathrm{g} / \mathrm{ml}$ and 0.25 to $1 \mu \mathrm{g} / \mathrm{ml}$, respectively. The highest observed ceftazidime-QPX7728 MIC for the mutants was $4 \mu \mathrm{g} / \mathrm{ml}$ (due to KPC-2::D179Y (KPC-31) mutation selected from KP1099, ceftazidime-avibactam MIC > $128 \mu \mathrm{g} / \mathrm{ml})$.

Additional studies are underway to directly assess the interaction of QPX7728 with various KPC variants that confer resistance to ceftazidime-avibactam. However, it appears that QPX7728 combined with multiple antibiotics is capable to overcome this resistance.

\section{QPX7728 Restores Potency of Meropenem Against Carbapenem-Resistant Acinetobacter baumannii (CRAB)}

A collection of 275 clinical isolates of highly carbapenem resistant Acinetobacter baumannii $\left(\mathrm{MIC}_{50} / \mathrm{MIC}_{90}\right.$ for meropenem of $64 />64 \mu \mathrm{g} / \mathrm{ml}$ ) was used to assess the enhancing effect of QPX7728 on meropenem potency (Nelson et al., 2020b). Combining meropenem with increasing concentrations of QPX7728 (1-16 $\mu \mathrm{g} / \mathrm{ml})$ resulted in a $>8-16$-fold increase in meropenem potency: $\mathrm{MIC}_{90}$ of meropenem decreased to 8 and $4 \mu \mathrm{g} / \mathrm{ml}$ by addition of QPX7728 at 4 and $8 \mu \mathrm{g} / \mathrm{ml}$, respectively (Figure 6D).

Numerous studies demonstrated that mutations in penicillin binding proteins, inactivation of porin genes and increased efflux are the most relevant non- $\beta$-lactamase-mediated mechanisms associated with reduced activity of meropenem against A. baumannii (Vila et al., 2007; Hawkey et al., 2018). We have found that the same mechanisms also increased the MIC values of meropenem/QPX7728 (Nelson et al., 2020b). Mutations in PBP3 that were mapped to the substrate-binding site appear to have the strongest impact on its potency increasing 4-8-fold the MIC $_{90}$ of meropenem/QPX7728. Nevertheless, MIC 90 for the strains that produced carbapenemases in the background of PBP3 mutations was still below the PK-PD breakpoint for meropenem $(8 \mu \mathrm{g} / \mathrm{ml})$. We also noted that when PBP3 mutations were absent, meropenem-QPX7728 MICs values were less than or equal to $8 \mu \mathrm{g} / \mathrm{ml}$ and in the absence of both PBP3 and efflux regulatory mutations, meropenem-QPX7728 MIC values were less than or equal to $4 \mu \mathrm{g} / \mathrm{ml}$. We believe that the systematic evaluation of resistance mechanisms and their impact on MIC will facilitate the future development of molecular tools that can be used to predict susceptibility and resistance to QPX7728 combinations.

The study described above (Nelson et al., 2020b) also allowed the determination of the TPC 90 of QPX7728 in combination with meropenem: it was less than or equal to $8 \mu \mathrm{g} / \mathrm{ml}$ for the majority 
TABLE 5 | In vitro activity ( $\mathrm{MIC}_{50} / \mathrm{MIC}_{90}$ and \% inhibited) of meropenem alone and combined with QPX7728 at 4 and $8 \mu \mathrm{g} / \mathrm{ml}$ and comparator BLI combination agents against carbapenem resistant strains of Enterobacterales according to the functional status of OmpK35/OmpF and OmpK36/OmpC and carbapenemase present.

\begin{tabular}{|c|c|c|c|c|c|}
\hline & $\begin{array}{c}\text { Meropenem- } \\
\text { QPX7728 at } \\
4 \mu \mathrm{g} / \mathrm{ml}\end{array}$ & $\begin{array}{c}\text { Meropenem- } \\
\text { QPX7728 at } \\
8 \mu \mathrm{g} / \mathrm{ml}\end{array}$ & $\begin{array}{c}\text { Meropnem- } \\
\text { vaborbactam at } \\
8 \mu \mathrm{g} / \mathrm{ml}\end{array}$ & $\begin{array}{c}\text { Ceftazidime- } \\
\text { avibactamat } \\
4 \mu \mathrm{g} / \mathrm{ml}\end{array}$ & $\begin{array}{l}\text { Imipenem- } \\
\text { relebactam at } \\
4 \mu \mathrm{g} / \mathrm{ml}\end{array}$ \\
\hline \multicolumn{6}{|l|}{ K. pneumonia KPC $(N=230)$} \\
\hline OmpK36-defective $(N=135)$ & $0.25 / 2$ & $0.125 / 0.5$ & $2 / 32$ & $4 / 16$ & $0.5 / 4$ \\
\hline$\%$ Inhibited & $94.8 \%$ & $100.0 \%$ & $72.6 \%$ & $88.1 \%$ & $70.4 \%$ \\
\hline OmpK36-functional $(N=95)$ & $\leq 0.06 / 0.125$ & $\leq 0.06 / \leq 0.06$ & $\leq 0.06 / 0.25$ & $2 / 8$ & $0.25 / 1$ \\
\hline$\%$ Inhibited* & $100.0 \%$ & $100.0 \%$ & $97.9 \%$ & $91.6 \%$ & $94.7 \%$ \\
\hline \multicolumn{6}{|l|}{$\operatorname{MBL}(N=177)$} \\
\hline OmpK36-defective $(N=42)$ & $4 / 32$ & $1 / 4$ & $>64 />64$ & $>64 />64$ & $>64 />64$ \\
\hline$\%$ Inhibited & $81.0 \%$ & $92.9 \%$ & $0.0 \%$ & $0.0 \%$ & $0.0 \%$ \\
\hline OmpK36-functional $(N=135)$ & $\leq 0.06 / 0.5$ & $\leq 0.06 / 0.125$ & $32>64$ & $>64 />64$ & $32>64$ \\
\hline$\%$ Inhibited & $99.3 \%$ & $99.3 \%$ & $20.0 \%$ & $2.2 \%$ & $3.7 \%$ \\
\hline
\end{tabular}

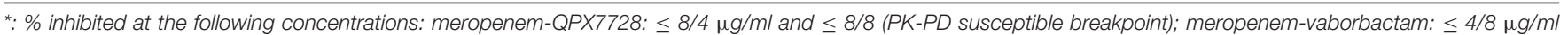
(FDA susceptible breakpoint); ceftazidime-avibactam: $\leq 8 / 4 \mu \mathrm{g} / \mathrm{ml}$ (FDA susceptible breakpoint); imipenem-relebactam: $\leq 1 / 4 \mu \mathrm{g} / \mathrm{ml}$ (FDA susceptible breakpoint).

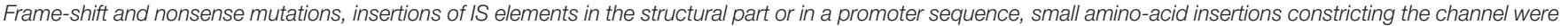
identified as mutations resulting in a defective protein. In the absence of these mutations, OmpK36 was assumed to be functional. Data from Nelson et al. (2020a).

TABLE 6 | The effect of KPC mutations selected with ceftazidime-avibactam on potency of QPX7728 in combination with ceftazidime, meropenem, and cefepime.

\begin{tabular}{|c|c|c|c|c|c|c|c|c|}
\hline Strain & B-lactamases & CAZ & CAZ+AVI & CAZ+QPX & MEM & MEM+QPX & FEP & FEP+QPX \\
\hline KP1074 & KPC-3, SHV-11, TEM-1/GD & $>128$ & 4 & 0.5 & 128 & $\leq 0.125$ & $>128$ & 0.5 \\
\hline KPM3200 & KPC-3::duplication of EAVI (aa\#278) & $>128$ & 64 & 0.5 & 64 & $\leq 0.125$ & $>128$ & 0.5 \\
\hline KPM3205 & KPC-3 ::duplication of KKDY (aa\#273) & $>128$ & 128 & 1 & 16 & $\leq 0.125$ & 128 & 0.5 \\
\hline KP1099 & KPC-2, SHV-11, SHV-12, CTX-M-14/GD & $>128$ & 2 & 1 & 128 & $\leq 0.125$ & $>128$ & 0.25 \\
\hline KPM3210 & KPC-2::D179N & $>128$ & 32 & 0.5 & 4 & $\leq 0.125$ & 128 & $\leq 0.125$ \\
\hline KPM3213 & KPC-2::D179Y (KPC-31) & $>128$ & $>128$ & 4 & 4 & $\leq 0.125$ & $>128$ & 0.25 \\
\hline KPM3214 & KPC-2::D179A & $>128$ & 128 & 2 & 2 & $\leq 0.125$ & 128 & 0.5 \\
\hline KPM3212 & deletion of ELNSAI at aa\#497 & $>128$ & 64 & 2 & 1 & $\leq 0.125$ & 128 & 0.5 \\
\hline KP1252 & KPC-3, TEM-1, SHV-11/FL & $>128$ & 8 & 1 & 64 & $\leq 0.125$ & $>128$ & 0.5 \\
\hline KPM3512 & KPC-3:: P174L & $>128$ & 32 & 2 & 8 & $\leq 0.125$ & 16 & $\leq 0.125$ \\
\hline KPM3511 & KPC-3::P174Q & $>128$ & 64 & 1 & 4 & 0.25 & 64 & 0.5 \\
\hline KP1317 & KPC-3, TEM-1, SHV-11/GD & $>128$ & 2 & 1 & $>128$ & $\leq 0.125$ & $>128$ & 0.5 \\
\hline KPM3517 & KPC-3::D179Y (KPC-31) & $>128$ & 64 & 1 & 128 & $\leq 0.125$ & $>128$ & 0.5 \\
\hline KPM3520 & Both WT and mutant KPC-3::duplication of 8aa (aa\#264-271) & $>128$ & 64 & 1 & 128 & 0.25 & $>128$ & 0.5 \\
\hline KPM3523 & Both WT and mutant KPC-3:: deletion of EL (aa\#241-242) & $>128$ & 32 & 1 & 128 & $\leq 0.125$ & 128 & 1 \\
\hline KP1500 & KPC-3, TEM-1, SHV-11, CTX-M-15 OXA-1 OXA-9/IS at -48 & $>128$ & 4 & 0.5 & $>128$ & $\leq 0.125$ & $>128$ & 0.5 \\
\hline KPM3546 & KPC-3::R164H & $>128$ & 32 & 1 & 128 & 0.25 & $>128$ & 0.5 \\
\hline KP1102 & KPC-3, TEM-1, SHV-11/GD & $>128$ & 4 & 1 & $>128$ & $\leq 0.125$ & $>128$ & 4 \\
\hline KPM3493 & KPC-3::duplication of EL at aa\#164 (KPC-25) & $>128$ & 16 & 2 & 1 & $\leq 0.125$ & 32 & 1 \\
\hline KPM3494 & KPC-3::R167S & $>128$ & 32 & 1 & 32 & $\leq 0.125$ & 128 & 1 \\
\hline KPM3495 & KPC-3::A175V & $>128$ & 32 & 1 & 32 & $\leq 0.125$ & $>128$ & 0.5 \\
\hline KP1246 & KPC-3, SHV-12, SHV-11/FL & $>128$ & 8 & 0.25 & 128 & $\leq 0.125$ & 128 & 0.25 \\
\hline KPM3191 & KPC-3:: DDK duplication at aa\#831 (KPC-29) & $>128$ & 64 & $\leq 0.125$ & 4 & $\leq 0.125$ & 64 & 0.25 \\
\hline KPM3192 & KPC-3:: D179Y (KPC-31) & $>128$ & 128 & 0.25 & 0.5 & $\leq 0.125$ & 32 & 0.25 \\
\hline
\end{tabular}

Avibactam and QPX7728 tested at $4 \mu \mathrm{g} / \mathrm{ml}$. bla KPC was detected in two different locations in the strain KP1317. Parent strains are in bold font. Data from Nelson et al. (2019).

of the subsets of strains stratified according to the presence of various intrinsic mechanisms, similar to what was observed in carbapenem-resistant Enterobacterales.

The plasma QPX7728 exposure associated with $8 \mu \mathrm{g} / \mathrm{ml}$ was found to be efficacious in animal models of infection (Sabet et al., 2020), and is being targeted in human dosage regimens during ongoing clinical development ${ }^{1}$. Thus, subsequent microbiological evaluation of QPX7728 in combination with IV antibiotics was performed using QPX7728 at a fixed concentration of $8 \mu \mathrm{g} / \mathrm{ml}$. We expect

\footnotetext{
${ }^{1}$ https://clinicaltrials.gov/ct2/show/NCT04380207
} 
TABLE 7 In vitro potency of various antibiotics alone and in combination with QPX7728 at $8 \mu \mathrm{g} / \mathrm{ml}$ and comparator agents against representative and challenge panels of clinical isolates of Pseudomonas aeruginosa.

The panel reflecting current MIC distribution for $\beta$-lactams and BL/BLI combinations $(N=500)$

\begin{tabular}{|c|c|c|c|c|c|c|c|c|c|}
\hline & MEM & QPX/MEM & TOL & TOL-TAZ & QPX/TOL & FEP & QPX/FEP & CAZ-AVI & PIP-TAZ \\
\hline $\mathrm{MIC}_{50}(\mu \mathrm{g} / \mathrm{ml})$ & 0.5 & 0.25 & 0.5 & 0.5 & 0.5 & 4 & 2 & 2 & 8 \\
\hline $\mathrm{MIC}_{90}(\mu \mathrm{g} / \mathrm{ml})$ & 16 & 8 & 4 & 4 & 1 & 32 & 8 & 8 & 128 \\
\hline \% Inhibited* & $84.8 \%$ & $91.6 \%$ & $90.4 \%$ & $91.8 \%$ & $97.6 \%$ & $74.4 \%$ & $91.2 \%$ & $92.2 \%$ & $71.6 \%$ \\
\hline
\end{tabular}

\begin{tabular}{|c|c|c|c|c|c|c|c|c|c|c|c|c|c|c|c|}
\hline All $(N=290)$ & & MEM & QPX/MEM & ATM & QPX/ATM & PIP & QPX/PIP & TOL & QPX/TOL & FEP & QPX/FEP & PIP-TAZ & TOL-TAZ & CAZ-AVI & IMP-REL \\
\hline & $\mathrm{MIC}_{50}$ & 16 & 8 & 32 & 16 & $>64$ & 16 & 4 & 1 & 32 & 8 & $>64$ & 4 & 8 & 4 \\
\hline & $\mathrm{MIC}_{90}$ & $>64$ & 64 & $>64$ & 64 & $>64$ & 64 & $>64$ & 64 & 64 & 64 & $>64$ & $>64$ & $>64$ & 64 \\
\hline \multirow[t]{3}{*}{ No MBL $(N=229)$} & $\%$ Inhibited $^{*}$ & 33.1 & 60.3 & 19.0 & 45.9 & 16.2 & 70.3 & 51.4 & 78.6 & 20.0 & 70.3 & 17.2 & 52.1 & 50.7 & 46.9 \\
\hline & $\mathrm{MIC}_{50}$ & 16 & 8 & 32 & 16 & $>64$ & 16 & 2 & 1 & 32 & 8 & $>64$ & 2 & 8 & 2 \\
\hline & $\mathrm{MIC}_{90}$ & 64 & 32 & $>64$ & 32 & $>64$ & 64 & $>64$ & 4 & 64 & 16 & $>64$ & $>64$ & 64 & 16 \\
\hline \multirow[t]{4}{*}{ MBL $(N=61)$} & $\%$ Inhibited $^{*}$ & 41.9 & 68.1 & 17.9 & 46.7 & 19.7 & 69.9 & 64.2 & 92.1 & 23.6 & 79.5 & 20.1 & 65.5 & 62.9 & 59.0 \\
\hline & $\mathrm{MIC}_{50}$ & $>64$ & 32 & 32 & 16 & $>64$ & 16 & $>64$ & 64 & 64 & 32 & $>64$ & $>64$ & $>64$ & $>64$ \\
\hline & $\mathrm{MIC}_{90}$ & $>64$ & $>64$ & $>64$ & 64 & $>64$ & 64 & $>64$ & $>64$ & 64 & 64 & $>64$ & $>64$ & $>64$ & $>64$ \\
\hline & $\%$ Inhibited* & 0.0 & 31.1 & 23.0 & 42.6 & 3.3 & 72.1 & 3.3 & 27.9 & 6.6 & 36.1 & 6.6 & 1.6 & 4.9 & 1.6 \\
\hline
\end{tabular}

MEM, meropenem; QPX, QPX7728; TOL, ceftolozane; TAZ, tazobactam; FEP, cefepime; CAZ-AVI, ceftazidime-avibactam; PIP-TAZ, piperacillin-tazobactam; tazobactam and avibactam were tested at fixed $4 \mu \mathrm{g} / \mathrm{ml}$. *. \% inhibited at the following concentrations: meropenem: $\leq 8 \mu \mathrm{g} / \mathrm{ml} ;$ QPX7728/meropenem: $\leq 8 \mu \mathrm{g} / \mathrm{m} /$ with QPX7728 at $8 \mu \mathrm{g} / \mathrm{ml} ; \mathrm{ceftolozane:} \leq 4 \mu \mathrm{g} / \mathrm{ml} ; \mathrm{QPX} 7728 / \mathrm{cefto} / \mathrm{ozane:} \leq 4 \mu \mathrm{g} / \mathrm{m} / \mathrm{with}$ QPX7728 at $8 \mu \mathrm{g} / \mathrm{ml} ;$ ceftolozane-tazobactam: $\leq 4 / 4 \mu \mathrm{g} / \mathrm{ml}$; cefepime: $\leq 8 \mu \mathrm{g} / \mathrm{ml}$; QPX7728/cefepime: $\leq 8 \mu \mathrm{g} / \mathrm{m} /$ with QPX7728 at $8 \mu \mathrm{g} / \mathrm{ml}$; ceftazidime-avibactam: $\leq 8 / 4 \mu \mathrm{g} / \mathrm{ml} ;$ piperacillin-tazobactam: $\leq 16 \mu \mathrm{g} / \mathrm{ml}$. Data from Lomovskaya et al. (2021). 
TABLE 8 | In vitro activity of QPX7728 in combination with oral $\beta$-lactams against Enterobacterales producing ESBLs and carbapenemases.

\begin{tabular}{|c|c|c|c|c|c|c|}
\hline \multirow[t]{2}{*}{ Organism } & \multicolumn{6}{|c|}{$\mathrm{MIC}_{50 / 90}(\mu \mathrm{g} / \mathrm{ml})$} \\
\hline & Cefpodoxime & Cefpodoxime-QPX7728 & Ceftibuten & Ceftibuten-QPX7728 & Tebipenem & Tebipenem-QPX7728 \\
\hline All Enterobacterales $(N=972)$ & $>64 />64$ & $1 / 16$ & $32 />64$ & $\leq 0.06 / 4$ & $0.25 />64$ & $\leq 0.06 / 1$ \\
\hline ESBLs, no CRE $(N=371)$ & $>64 />64$ & $0.5 / 4$ & $8 />64$ & $\leq 0.06 / 1$ & $\leq 0.06 / 0.5$ & $\leq 0.06 / 0.125$ \\
\hline CRE KPC $(N=292)$ & $>64 />64$ & $2 / 8$ & $16 />64$ & $0.125 / 0.5$ & $64 />64$ & $\leq 0.06 / 2$ \\
\hline CRE OXA-48 $(N=47)$ & $>64 />64$ & $1 / 8$ & $64 />64$ & $0.125 / 1$ & $32 / 64$ & $0.25 / 0.5$ \\
\hline CRE MBL $(N=226)$ & $>64 />64$ & $>64 />64$ & $>64 />64$ & $2 />64$ & $32 />64$ & $0.125 / 32$ \\
\hline Non-CP CRE & $>64 />64$ & $>64 />64$ & $>64 />64$ & $2 />64$ & $8 / 16$ & $1 / 4$ \\
\hline
\end{tabular}

Non-CP CRE, no carbapenemasee-producing CRE; QPX7728 tested at $4 \mu \mathrm{g} / \mathrm{ml}$. Data from Rubio-Aparicio et al. (2019).

this concentration of QPX7728 will be adopted for future in vitro susceptibility testing using various manual, semiautomatic and automatic systems.

\section{QPX7728 Enhances Potency of Multiple $\beta$-Lactam Antibiotics Against Pseudomonas aeruginosa}

The study described by Lomovskaya et al. (2021) investigated the impact of QPX7728 on potency of meropenem, cefepime and ceftolozane against two panels of $P$. aeruginosa. The first panel consisted of 500 isolates that were selected to mirror MIC distributions of $\beta$-lactams and BL/BLI combinations observed in recent global surveillance studies. QPX7728 shifted > 90\% of these isolates below susceptibility breakpoint of $8 \mu \mathrm{g} / \mathrm{ml}$ for meropenem and cefepime or $4 \mu \mathrm{g} / \mathrm{ml}$ for ceftolozane (Table 7). In agreement with surveillance reports (Sader et al., 2020), ceftolozane-tazobactam and ceftazidime-avibactam were also highly potent, inhibiting $>90 \%$ of these isolates at their susceptibility breakpoints.

The enhancement of potency of multiple $\beta$-lactams by QPX7728 was also observed against the second panel of strains $(\mathrm{N}=290)$ consisting of meropenem or ceftolozanetazobactam non-susceptible or ceftazidime-avibactam resistant isolates. QPX7728 in combination with meropenem, ceftolozane, cefepime and piperacillin inhibited a higher percentage strains at the susceptibility breakpoint for the partner $\beta$-lactam alone compared to clinically used BL/BLI combinations. In general, the highest activity was observed for QPX7728-ceftolozane, with $78.6 \%$ of isolates inhibited at $4 \mu \mathrm{g} / \mathrm{ml}$ of ceftolozane. $70.3 \%$ of isolates were shifted by QPX7728 at or below 8 or $16 \mu \mathrm{g} / \mathrm{ml}$ for cefepime and piperacillin, respectively, and $60.3 \%$ of isolates were inhibited by QPX7728-meropenem at MIC of $\leq 8 \mu \mathrm{g} / \mathrm{ml}$ (Table 7 ).

The result from this study is that the activity of QPX7728/ $\beta$ lactam combinations will be determined by multiple factors. First, the liability of a specific $\beta$-lactam to hydrolysis of a particular $\beta$-lactamase, i.e., if the $\beta$-lactamase is a cephaloporinase vs. carbapenemase vs. penicillinase. Second, the inhibitory potency of QPX7728 for a particular $\beta$-lactamase. Finally, the impact of non- $\beta$-lactamase mediated $\beta$-lactam resistance mechanisms such as increased efflux by MexAB-OprM or inactivation of OprD on both the inhibitor and $\beta$-lactam will also impact activity (Lomovskaya et al., 2021).

Data obtained in biochemical experiments indicate that QPX7728 inhibits serine $\beta$-lactamases with higher potency than metallo enzymes, consistent with higher activity of QPX7728-meropenem, QPX7728-ceftolozane and QPX7728cefepime against MBL-negative than MBL-positive strains. Data from MIC experiments using cloned $\beta$-lactamases indicate that potentiation with QPX7728 is more effective with antibiotics that are less susceptible to $\beta$-lactamase-mediated hydrolysis. This is consistent with the higher potency of QPX7728piperacillin (piperacillin appears to be less affected by MBLmediated hydrolysis compared to ceftolozane, cefepime and meropenem) combination against the MBL-positive strains compared to other QPX7728 combinations. As expected, the potency of QPX-aztreonam does not depend on the presence or absence of MBLs as aztreonam is not hydrolyzed by these enzymes.

Studies with isogenic strains demonstrated that various combinations of non- $\beta$-lactamase-mediated intrinsic mechanisms such as increased efflux by MexAB-OprM or inactivation of a carbapenem-specific porin OprD may raise the MICs of some $\beta$-lactams to or above their susceptibility breakpoints (Lomovskaya et al., 2021). The combination of QPX7728 plus ceftolozane is the most potent combination against MBL-negative strains of Pseudomonas aeruginosa since neither drug is a substrate of efflux nor is affected by inactivation of OprD. In contrast, meropenem MICs can be raised to or above the PK-PD breakpoint of $8 \mu \mathrm{g} / \mathrm{ml}$ when overexpression of the MexAB-OprM efflux pump is combined with inactivation of OprD. These observations are important for the potential future use of $\beta$-lactamase inhibitors; different $\beta$-lactams can be chosen as companion antibiotics for QPX7728 to achieve the optimal therapeutic effect. Co-administration of QPX7728 with a specific $\beta$-lactam will depend on resistance mechanisms identified in the specific pathogen. Undoubtedly, timely selection of the best companion $\beta$-lactam at the individual strain/patient level requires availability of efficient susceptibility testing methods and can be facilitated by the development of molecular tools based on deep understanding of the impact of numerous resistance mechanisms on antibiotics potency. This strategy might give physicians more options when managing infections due to highly resistant organisms, particularly in patients with limited choices. 


\section{QPX7728 Enhances the Potency of Oral $\beta$-Lactam Antibiotics Against Enterobacterales Producing ESBLs or Carbapenemases}

QPX7728 has 43-53\% oral bioavailability in rats at doses of $30-100 \mathrm{mg} / \mathrm{kg}$ (Hecker et al., 2020) and an oral formulation is being developed for oral administration. Using a large panel of ESBL and carbapenemase-producing strains of Enterobacterales we demonstrated (Rubio-Aparicio et al., 2019) that QPX7728 significantly enhanced the potency of multiple $\beta$-lactams against these strains (Table 8).

\section{CONCLUSION}

In conclusion, QPX7728 is a novel ultra-broad-spectrum $\beta$ lactamase inhibitor with the broadest spectrum of inhibition reported up to date in a single BLI molecule. The ability to inhibit metallo enzymes did not come at the expense of reduced spectrum or potency against serine $\beta$-lactamases. On the contrary, the spectrum of inhibition of serine enzymes by QPX7728 was also expanded compared to that of the clinically available BLIs - it has a potent activity against serine enzymes that historically have been difficult to inhibit such as class D carbapenemases from A. baumannii (e.g., OXA-23). Importantly it is minimally affected by general intrinsic resistance mechanisms such as efflux and porin mutations. QPX7728 combinations with several IV $\beta$-lactam antibiotics showed high potency against strains of Enterobacterales, Acinetobacter baumannii and Pseudomonas aeruginosa that are resistant to other recently approved IV $\beta$-lactam-BLI combinations, e.g.,

\section{REFERENCES}

Barnes, M. D., Winkler, M. L., Taracila, M. A., Page, M. G., Desarbre, E., Kreiswirth, B. N., et al. (2017). Klebsiella pneumoniae carbapenemase-2 (KPC2), substitutions at ambler position Asp179, and resistance to ceftazidimeavibactam: unique antibiotic-resistant phenotypes emerge from beta-lactamase protein engineering. MBio 8, e00528-17.

Bush, K. (2018). Past and present perspectives on beta-lactamases. Antimicrob. Agents Chemother. 62, 1-20.

Bush, K., and Bradford, P. A. (2019). Interplay between beta-lactamases and new beta-lactamase inhibitors. Nat. Rev. Microbiol. 17, 295-306. doi: 10.1038/ s41579-019-0159-8

Calzi, A., Grignolo, S., Caviglia, I., Calevo, M. G., Losurdo, G., Piaggio, G., et al. (2016). Resistance to oral antibiotics in 4569 Gram-negative rods isolated from urinary tract infection in children. Eur. J. Pediatr. 175, 1219-1225. doi: 10.1007/ s00431-016-2763-1

Castanheira, M., Doyle, T. B., Smith, C. J., Mendes, R. E., and Sader, H. S. (2019). Combination of MexAB-OprM overexpression and mutations in efflux regulators, $\mathrm{PBPs}$ and chaperone proteins is responsible for ceftazidime/avibactam resistance in Pseudomonas aeruginosa clinical isolates from US hospitals. J. Antimicrob. Chemother. 74, 2588-2595. doi: 10.1093/jac/ dkz243

CDC (2019). Antibiotic Resistance Threats in the United States, 2019. Atlanta, GA: U.S. Department of Health and Human Services, CDC.

Chalhoub, H., Sáenz, Y., Nichols, W. W., Tulkens, P. M., and Van Bambeke, F. (2018). Loss of activity of ceftazidime-avibactam due to MexAB-OprM efflux and overproduction of AmpC cephalosporinase in Pseudomonas aeruginosa isolated from patients suffering from cystic fibrosis. Int. ceftazidime-avibactam, ceftolozane-tazobactam, meropenemvaborbactam and imipenem-relebactam. Due to its broad $\beta$-lactamase inhibition spectrum and the ability to potentiate multiple antibiotics against multiple target pathogens, QPX7728 is suited perfectly for a stand-alone BLI strategy, where it can be combined with multiple different $\beta$-lactams, with the goal of providing more optionality for both treatment and stewardship efforts. QPX7728 can be delivered orally, and in combination with oral $\beta$-lactams has promising activity against ESBL and carbapenemase-producing strains of Enterobacterales ensuring that ultra-broad-spectrum $\beta$-lactamase inhibition spectrum and other features of QPX7728 could be applied to both IV and oral QPX7728-based products. Clinical development of QPX7728 has been initiated.

\section{AUTHOR CONTRIBUTIONS}

OL wrote the manuscript with critical feedback from all authors. MT, JL, and MD contributed to the final version of the manuscript. All authors are members of the team that is developing QPX7728 and contributed to the results described in this overview.

\section{FUNDING}

This project has been funded in whole or in part with Federal funds from the Department of Health and Human Services; Office of the Assistant Secretary for Preparedness and Response; Biomedical Advanced Research and Development Authority (BARDA), under OTA number HHSO100201600026C.
J. Antimicrob. Agents 52, 697-701. doi: 10.1016/j.ijantimicag.2018. 07.027

Chevalier, S., Bouffartigues, E., Bodilis, J., Maillot, O., Lesouhaitier, O., Feuilloley, M. G. J., et al. (2017). Structure, function and regulation of Pseudomonas aeruginosa porins. FEMS Microbiol. Rev. 41, 698-722. doi: 10.1093/femsre/ fux020

Coyne, S., Courvalin, P., and Périchon, B. (2011). Efflux-mediated antibiotic resistance in Acinetobacter spp. Antimicrob. Agents Chemother. 55, 947-953.

Critchley, I. A., Cotroneo, N., Pucci, M. J., and Mendes, R. (2019). The burden of antimicrobial resistance among urinary tract isolates of Escherichia coli in the United States in 2017. PLoS One 14:e0220265. doi: 10.1371/journal.pone. 0220265

Ehmann, D. E., Jahic, H., Ross, P. L., Gu, R. F., Hu, J., Durand-Reville, T. F., et al. (2013). Kinetics of avibactam inhibition against Class A, C, and D betalactamases. J. Biol. Chem. 288, 27960-27971. doi: 10.1074/jbc.m113.485979

El Zowalaty, M. E., Al Thani, A. A., Webster, T. J., El Zowalaty, A. E., Schweizer, H. P., Nasrallah, G. K., et al. (2015). Pseudomonas aeruginosa: arsenal of resistance mechanisms, decades of changing resistance profiles, and future antimicrobial therapies. Future Microbiol. 10, 1683-1706. doi: 10.2217/fmb. 15.48

Galleni, M., Lamotte-Brasseur, J., Rossolini, G. M., Spencer, J., Dideberg, O., Frere, J. M., et al. (2001). Standard numbering scheme for class B beta-lactamases. Antimicrob. Agents Chemother. 45, 660-663. doi: 10.1128/aac.45.3.660-663. 2001

Gomez-Simmonds, A., Stump, S., Giddins, M. J., Annavajhala, M. K., and Uhlemann, A. C. (2018). Clonal Background, resistance gene profile, and porin gene mutations modulate in vitro susceptibility to imipenem-relebactam in diverse Enterobacteriaceae. Antimicrob. Agents Chemother. 62, e00573-18. 
Hawkey, J., Ascher, D. B., Judd, L. M., Wick, R. R., Kostoulias, X., Cleland, H., et al. (2018). Evolution of carbapenem resistance in Acinetobacter baumannii during a prolonged infection. Microb. Genom. 4:e000165.

Hecker, S. J., Reddy, K. R., Lomovskaya, O., Griffith, D. C., Rubio-Aparicio, D., Nelson, K., et al. (2020). Discovery of cyclic boronic acid QPX7728, an ultrabroad-spectrum inhibitor of serine and metallo beta-lactamases. J. Med. Chem. 63, 7491-7507. doi: 10.1021/acs.jmedchem.1029b01976

Hecker, S. J., Reddy, K. R., Totrov, M., Hirst, G. C., Lomovskaya, O., Griffith, D. C., et al. (2015). Discovery of a cyclic boronic acid beta-lactamase inhibitor (RPX7009) with utility vs Class A Serine Carbapenemases. J.Med. Chem. 58, 3682-3692. doi: 10.1021/acs.jmedchem.5b00127

Ho, S., Nguyen, L., Trinh, T., and Macdougall, C. (2019). Recognizing and overcoming resistance to new beta-lactam/beta-lactamase inhibitor combinations. Curr. Infect. Dis. Rep. 21:39.

Kuti, J. L., Dandekar, P. K., Nightingale, C. H., and Nicolau, D. P. (2003). Use of monte carlo simulation to design an optimized pharmacodynamic dosing strategy for meropenem. J. Clin. Pharmacol. 43, 1116-1123. doi: 10.1177/ 0091270003257225

Lee, L. S., Kinzig-Schippers, M., Nafziger, A. N., Ma, L., Sorgel, F., Jones, R. N., et al. (2010). Comparison of 30-min and 3-h infusion regimens for imipenem/cilastatin and for meropenem evaluated by Monte Carlo simulation. Diagn. Microbiol. Infect. Dis. 68, 251-258. doi: 10.1016/j.diagmicrobio.2010.06. 012

Li, X.-Z., Plésiat, P., and Nikaido, H. (2015). The challenge of efflux-mediated antibiotic resistance in gram-negative bacteria. Clin. Microbiol. Rev. 28, 337418. doi: $10.1128 / \mathrm{cmr} .00117-14$

Li, X.-Z., Zhang, L., Srikumar, R., and Poole, K. (1998). $\beta$-lactamase inhibitors are substrates for the multidrug efflux pumps of Pseudomonas aeruginosa. Antimicrob. Agents Chemother. 42, 399-403. doi: 10.1128/aac.42.2.399

Lister, P. D., Wolter, D. J., and Hanson, N. D. (2009). Antibacterialresistant Pseudomonas aeruginosa: clinical impact and complex regulation of chromosomally encoded resistance mechanisms. Clin. Microbiol. Rev. 22, 582-610. doi: 10.1128/cmr.00040-09

Livermore, D. M., Warner, M., Jamrozy, D., Mushtaq, S., Nichols, W. W., Mustafa, N., et al. (2015). In vitro selection of ceftazidime-avibactam resistance in Enterobacteriaceae with KPC-3 carbapenemase. Antimicrob. Agents Chemother. 59, 5324-5330. doi: 10.1128/aac.00678-15

Lomovskaya, O., Nelson, K., Rubio-Aparicio, D., Tsivkovski, R., Sun, D., and Dudley, M. N. (2020a). Impact of intrinsic resistance mechanisms on potency of QPX7728, a new ultrabroad-spectrum beta-lactamase inhibitor of serine and metallo-beta-lactamases in Enterobacteriaceae, Pseudomonas aeruginosa, and Acinetobacter baumannii. Antimicrob. Agents Chemother. 64, e00552-20.

Lomovskaya, O., Rubio-Aparicio, D., Nelson, K., Sun, D., Tsivkovski, R., Castanheira, M., et al. (2021). In vitro activity of the ultra-broad-spectrum beta-lactamase inhibitor QPX7728 in combination with multiple beta-lactam antibiotics against Pseudomonas aeruginosa. Antimicrob. Agents Chemother 65, e00210-21.

Lomovskaya, O., Sun, D., Rubio-Aparicio, D., Nelson, K., Tsivkovski, R., Griffith, D. C., et al. (2017). Vaborbactam: spectrum of beta-lactamase inhibition and impact of resistance mechanisms on activity in Enterobacteriaceae. Antimicrob. Agents Chemother. 61, e01443-17.

Lomovskaya, O., Tsivkovski, R., Nelson, K., Rubio-Aparicio, D., Sun, D., Totrov, M., et al. (2020b). Spectrum of beta-lactamase inhibition by the cyclic boronate QPX7728, an ultrabroad-spectrum beta-lactamase inhibitor of serine and metallo-beta-lactamases: enhancement of activity of multiple antibiotics against isogenic strains expressing single beta-lactamases. Antimicrob. Agents Chemother. 64, e00212-e00220.

Mazzariol, A., Bazaj, A., and Cornaglia, G. (2017). Multi-drug-resistant Gramnegative bacteria causing urinary tract infections: a review. J. Chemother. 29, 2-9. doi: 10.1080/1120009x.2017.1380395

Nelson, K., Hemarajata, P., Sun, D., Rubio-Aparicio, D., Tsivkovski, R., Yang, S., et al. (2017). Resistance to ceftazidime-avibactam is due to transposition of KPC in a porin-deficient strain of Klebsiella pneumoniae with increased efflux activity. Antimicrob. Agents Chemother. 61, e00989-17.

Nelson, K., Rubio-Aparicio, D., and Lomovskaya, O. (2019). “QPX7728: resistance selection, prevention, and molecular mechanisms in mutants of KPCproducing Klebsiella pneumoniae," in Proceedings of the ASM-ESCMID. Abstract \#44, (Boston, MA).
Nelson, K., Rubio-Aparicio, D., Sun, D., Dudley, M., and Lomovskaya, O. (2020a). In vitro activity of the ultra-broad-spectrum beta-lactamase inhibitor QPX7728 against carbapenem-resistant enterobacterales (cre) with varying intrinsic and acquired resistance mechanisms. Antimicrob. Agents Chemother. 64, e00757-20. doi: 10.1128/AAC.00757-00720

Nelson, K., Rubio-Aparicio, D., Tsivkovski, R., Sun, D., Totrov, M., Dudley, M., et al. (2020b). In vitroactivity of the ultra-broad-spectrum betalactamase inhibitor QPX7728 in combination with meropenem against clinical isolates of carbapenem-resistant Acinetobacter baumannii. Antimicrob. Agents Chemother. 64, e01406-e01420.

Ness, S., Martin, R., Kindler, A. M., Paetzel, M., Gold, M., Jensen, S. E., et al. (2000). Structure-based design guides the improved efficacy of deacylation transition state analogue inhibitors of TEM-1 beta-Lactamase(). Biochemistry 39, 5312-5321. doi: 10.1021/bi992505b

Nikaido, H. (2003). Molecular basis of bacterial outer membrane permeability revisited. Microbiol. Mol. Biol. Rev. 67, 593-656. doi: 10.1128/mmbr.67.4.593656.2003

Nori, P., Szymczak, W., Puius, Y., Sharma, A., Cowman, K., Gialanella, P., et al. (2020). Emerging Co-Pathogens: New Delhi Metallo-beta-lactamase producing Enterobacterales Infections in New York City COVID-19 Patients. Int. J. Antimicrob. Agents 56:106179. doi: 10.1016/j.ijantimicag.2020.106179

Ochs, M., Lu, C.-D., Hancock, R., and Abdelal, A. (1999). Amino acid-mediated induction of the basic amino acid-specific outer membrane porin OprD from Pseudomonas aeruginosa. J. Bacteriol. 181, 5426-5432. doi: 10.1128/jb.181.17. 5426-5432.1999

Pages, J. M., James, C. E., and Winterhalter, M. (2008). The porin and the permeating antibiotic: a selective diffusion barrier in Gram-negative bacteria. Nat. Rev. Microbiol. 6, 893-903. doi: 10.1038/nrmicro1994

Pages, J.-M., Lavigne, J.-P., Leflon-Guibout, V., Marcon, E., Bert, F., Noussair, L., et al. (2009). Efflux Pump, the masked side of B-Lactam resistance in Klebsiella pneumoniae clinical isolates. PLoS One 4:e4817. doi: 10.1371/journal.pone. 0004817

Papp-Wallace, K. M. (2019). The latest advances in beta-lactam/beta-lactamase inhibitor combinations for the treatment of Gram-negative bacterial infections. Expert Opin. Pharmacother. 20, 2169-2184. doi: 10.1080/14656566.2019. 1660772

Papp-Wallace, K. M., Barnes, M. D., Alsop, J., Taracila, M. A., Bethel, C. R., Becka, S. A., et al. (2018). Relebactam is a potent inhibitor of the KPC-2 beta-lactamase and restores imipenem susceptibility in KPC-producing Enterobacteriaceae. Antimicrob. Agents Chemother. 62, e00174-18.

Pemberton, O. A., Tsivkovski, R., Totrov, M., Lomovskaya, O., and Chen, Y. (2020). Structural basis and binding kinetics of vaborbactam in class A $\beta$-lactamase inhibition. Antimicrob. Agents Chemother. 64, e00398-20.

Porretta, A. D., Baggiani, A., Arzilli, G., Casigliani, V., Mariotti, T., Mariottini, F., et al. (2020). Increased risk of acquisition of New Delhi metallo-beta-lactamaseproducing carbapenem-resistant enterobacterales (NDM-CRE) among a cohort of COVID-19 patients in a teaching hospital in tuscany, Italy. Pathogens 9:635. doi: 10.3390/pathogens 9080635

Reddy, R., Glinka, T., Totrov, M., and Hecker, S. (2014). Boronic Acid Derivatives and Therapeutic Uses Thereof. Patent WO2014107535A1.

Reddy, R., Glinka, T., Totrov, M., Hecker, S., and Lomovskaya, O. (2016). Boronic Acid Derivatives and Therapeutic Uses Thereof. Patent WO2016003929A1.

Rojas, L. J., Taracila, M. A., Papp-Wallace, K. M., Bethel, C. R., Caselli, E., Romagnoli, C., et al. (2016). Boronic acid transition state inhibitors active against KPC and other class A beta-lactamases: structure-activity relationships as a guide to inhibitor design. Antimicrob. Agents Chemother. 60, 1751-1759. doi: 10.1128/aac.02641-15

Rubio-Aparicio, D., Nelson, K., Griffith, D., Dudley, M., and Lomovskaya, O. (2019). "QPX7728: in vitro activity in combination with oral beta-lactam antibiotics against Enterobacteriaceae (ENT)," in Proceedings of the ASM Microbe AAR-710, (San Francisco, CA).

Sabet, M., Tarazi, Z., and Griffith, D. C. (2020). In vivo activity of QPX7728, an ultrabroad-spectrum beta-lactamase inhibitor, in combination with betalactams against carbapenem-resistant Klebsiella pneumoniae. Antimicrob. Agents Chemother. 64, e01267-20.

Sader, H. S., Flamm, R. K., Carvalhaes, C. G., and Castanheira, M. (2020). Comparison of ceftazidime-avibactam and ceftolozane-tazobactam in vitro activities when tested against gram-negative bacteria isolated from patients 
hospitalized with pneumonia in United States medical centers (2017-2018). Diagn. Microbiol. Infect. Dis. 96:114833. doi: 10.1016/j.diagmicrobio.2019.05. 005

Shields, R. K., Chen, L., Cheng, S., Chavda, K. D., Press, E. G., Snyder, A., et al. (2017). Emergence of ceftazidime-avibactam resistance due to plasmid-borne blaKPC-3 mutations during treatment of carbapenemresistant Klebsiella pneumoniae infections. Antimicrob. Agents Chemother. 61, e02097-16.

Shields, R. K., Clancy, C. J., Hao, B., Chen, L., Press, E. G., Iovine, N. M., et al. (2015). Effects of Klebsiella pneumoniae carbapenemase subtypes, extended-spectrum beta-lactamases, and porin mutations on the in vitro activity of ceftazidime-avibactam against carbapenem-resistant K. pneumoniae. Antimicrob. Agents Chemother. 59, 5793-5797. doi: 10.1128/aac.00548-15

Sommer, L. M., Johansen, H. K., and Molin, S. (2020). Antibiotic resistance in Pseudomonas aeruginosa and adaptation to complex dynamic environments. Microbial. Genomics 6:e000370.

Srikumar, R., Tsang, E., and Poole, K. (1999). Contribution of the MexABOprM multidrug e? ux system to the $\beta$-lactam resistance of penicillin-binding protein and $\beta$-lactamase-derepressed mutants of Pseudomonas aeruginosa. J. Antimicrob. Chemother. 44, 537-540. doi: 10.1093/jac/44.4.537

Talbot, G. H., Jezek, A., Murray, B. E., Jones, R. N., Ebright, R. H., Nau, G. J., et al. (2019). The infectious diseases society of America's 10 x '20 Initiative (10 New Systemic Antibacterial Agents US Food and Drug Administration Approved by 2020): Is 20 x '20 a Possibility? Clin. Infect. Dis. 69, 1-11. doi: 10.1093/cid/ ciz089

Tooke, C. L., Hinchliffe, P., Krajnc, A., Mulholland, A. J., Brem, J., Schofield, C. J., et al. (2020). Cyclic boronates as versatile scaffolds for KPC-2 $\beta$-lactamase inhibition. RSC Med. Chem. 11, 491-496. doi: 10.1039/c9md00557a

Trautner, B. W. (2018). Fluoroquinolones for urinary tract infection and withinhousehold spread of resistant Enterobacteriaceae: the smoking gun. Clin. Microbiol. Infect. 24, 929-930. doi: 10.1016/j.cmi.2018.03.038

Tsivkovski, R., and Lomovskaya, O. (2019). Biochemical activity of vaborbactam. Antimicrob. Agents Chemother. 64, e01935-19.
Tsivkovski, R., and Lomovskaya, O. (2020). Potency of vaborbactam is less affected than avibactam in strains producing KPC-2 mutations that confer resistance to ceftazidime-avibactam. Antimicrob. Agents Chemother. 64, e10936-19.

Tsivkovski, R., Totrov, M., and Lomovskaya, O. (2020). biochemical characterization of QPX7728, a new ultrabroad-spectrum beta-lactamase inhibitor of serine and metallo-beta-lactamases. Antimicrob. Agents Chemother. 64, e00130-20.

Vila, J., Martí, S., and Sánchez-Céspedes, J. (2007). Porins, efflux pumps and multidrug resistance in Acinetobacter baumannii. J. Antimicrob. Chemother. 59, 1210-1215. doi: 10.1093/jac/dkl509

WHO (2017). Global Priority List of Antibiotic-Resistant Bacteria to Guide Research, Discovery, and Development of New Antibiotics. Geneva: World Health Organization.

Wilson, W. R., Kline, E. G., Jones, C. E., Morder, K. T., Mettus, R. T., Doi, Y., et al. (2019). Effects of KPC variant and porin genotype on the in vitro activity of meropenem-vaborbactam against carbapenem-resistant Enterobacteriaceae. Antimicrob. Agents Chemother. 63, e02048-18.

Conflict of Interest: OL, RT, DS, RR, SH, DG, JL, and MD are employees and shareholders of Qpex Biopharma that is developing QPX7728. MT is an employee of Molsoft, LLC.

The remaining author declares that the research was conducted in the absence of any commercial or financial relationships that could be construed as a potential conflict of interest.

Copyright (c) 2021 Lomovskaya, Tsivkovski, Sun, Reddy, Totrov, Hecker, Griffith, Loutit and Dudley. This is an open-access article distributed under the terms of the Creative Commons Attribution License (CC BY). The use, distribution or reproduction in other forums is permitted, provided the original author(s) and the copyright owner(s) are credited and that the original publication in this journal is cited, in accordance with accepted academic practice. No use, distribution or reproduction is permitted which does not comply with these terms. 